\title{
Gametocyte carriage in uncomplicated Plasmodium falciparum malaria following treatment with artemisinin combination therapy: a systematic review and meta- analysis of individual patient data
}

WWARN Gametocyte Study Group ${ }^{1,2}$

\begin{abstract}
Background: Gametocytes are responsible for transmission of malaria from human to mosquito. Artemisinin combination therapy (ACT) reduces post-treatment gametocyte carriage, dependent upon host, parasite and pharmacodynamic factors. The gametocytocidal properties of antimalarial drugs are important for malaria elimination efforts. An individual patient clinical data meta-analysis was undertaken to identify the determinants of gametocyte carriage and the comparative effects of four ACTs: artemether-lumefantrine (AL), artesunate/amodiaquine (AS-AQ), artesunate/mefloquine (AS-MQ), and dihydroartemisinin-piperaquine (DP).

Methods: Factors associated with gametocytaemia prior to, and following, ACT treatment were identified in multivariable logistic or Cox regression analysis with random effects. All relevant studies were identified through a systematic review of PubMed. Risk of bias was evaluated based on study design, methodology, and missing data.

Results: The systematic review identified 169 published and 9 unpublished studies, 126 of which were shared with the WorldWide Antimalarial Resistance Network (WWARN) and 121 trials including 48,840 patients were included in the analysis. Prevalence of gametocytaemia by microscopy at enrolment was $12.1 \%(5887 / 48,589)$, and increased with decreasing age, decreasing asexual parasite density and decreasing haemoglobin concentration, and was higher in patients without fever at presentation. After ACT treatment, gametocytaemia appeared in $1.9 \%(95 \% \mathrm{Cl}, 1.7-2.1)$ of patients. The appearance of gametocytaemia was lowest after AS-MQ and AL and significantly higher after DP (adjusted hazard ratio (AHR), 2.03; $95 \% \mathrm{Cl}, 1.24-3.12 ; P=0.005$ compared to AL) and AS-AQ fixed dose combination (FDC) (AHR, 4.01; $95 \% \mathrm{Cl}, 2.40-6.72 ; P<0.001$ compared to AL). Among individuals who had gametocytaemia before treatment, gametocytaemia clearance was significantly faster with AS-MQ (AHR, 1.26; $95 \% \mathrm{Cl}, 1.00-1.60 ; P=0.054)$ and slower with DP (AHR, 0.74; $95 \% \mathrm{Cl}, 0.63-0.88 ; P=0.001)$ compared to AL. Both recrudescent (adjusted odds ratio (AOR), 9.05; $95 \% \mathrm{Cl}, 3.74-21.90 ; P<0.001)$ and new (AOR, 3.03; $95 \% \mathrm{Cl}, 1.66-5.54 ; P<0.001)$ infections with asexual-stage parasites were strongly associated with development of gametocytaemia after day 7 .

(Continued on next page)
\end{abstract}

\footnotetext{
Correspondence: teun.bousema@radboudumc.nl;

kasia.stepniewska@wwarn.org

'Department of Medical Microbiology 268, Radboud University Medical

Center, PO Box 9101, 6500HB Nijmegen, the Netherlands

${ }^{2}$ WorldWide Antimalarial Resistance Network (WWARN), Centre for Tropical

Medicine and Global Health, Churchill Hospital, CCVTM, University of Oxford,

Old Road, Oxford OX3 7LE, UK
} 
(Continued from previous page)

Conclusions: AS-MQ and AL are more effective than DP and AS-AQ FDC in preventing gametocytaemia shortly after treatment, suggesting that the non-artemisinin partner drug or the timing of artemisinin dosing are important determinants of post-treatment gametocyte dynamics.

Keywords: Malaria, Plasmodium falciparum, Drug resistance, Gametocyte

\section{Background}

Malaria remains a leading cause of morbidity and mortality in endemic countries, with an estimated 584,000 deaths and 198 million clinical cases of malaria globally in 2013 [1]. Considerable progress has been made in the last decade in reducing the burden of malaria by wide-scale deployment of insecticide-treated nets and efficacious artemisinin combination therapy (ACT) as first-line antimalarial treatment [2]. To maintain these gains and further move towards malaria elimination, a specific focus on malaria reducing interventions is needed [3]. The transmission of malaria to mosquitoes depends on mature sexual stage parasites, gametocytes, in the human peripheral blood. Plasmodium falciparum gametocytaemia has been associated with asexual parasite densities, the duration of malaria symptoms, anaemia and immunity $[4,5]$. A large fraction of gametocyte-positive individuals are asymptomatic and the contribution of this asymptomatic reservoir to onward malaria transmission is considerable in many endemic settings [6]. As a consequence, efforts to reduce malaria transmission by antimalarial treatment depend for a large extent on the proportion of malariainfected individuals that receive treatment [7]. Upon initiation of treatment, gametocytes may persist for several weeks after the clearance of asexual parasites with their longevity and infectivity depending on the treatment dispensed [8, 9], dosing [10] and host immunity [5].

ACT is now recommended universally for the treatment of uncomplicated falciparum malaria. Artemisinins are highly effective against the pathogenic asexual parasite stages [11] and immature gametocytes [12, 13], resulting in a substantial reduction of post-treatment malaria transmission compared to non-artemisinin drugs $[9,14,15]$. The wide-scale deployment of ACTs has been associated with substantial reductions in disease burden across a range of endemic settings [16, 17]. Nevertheless, the transmission reducing effects of ACT may be incomplete because of limited efficacy of artemisinins against mature gametocytes, permitting residual transmission in the first weeks after treatment $[9,15]$. Moreover, differences in artemisinin dosing, timing and partner drugs affect their gametocytocidal properties $[18,19]$.

Because gametocytes are only detected in a fraction of patients by microscopy, individual trials are often insufficiently powered to compare gametocytocidal properties between ACTs or disentangle host and parasite factors that influence gametocyte dynamics. To address this, a pooled analysis of individual-level patient data was undertaken in patients before and after treatment with artemether-lumefantrine (AL), artesunate-amodiaquine (AS-AQ), artesunate-mefloquine (AS-MQ), and dihydroartemisinin-piperaquine (DP).

\section{Methods}

Data pooling

A search was conducted in PubMed in September 2014 to identify all antimalarial clinical trials published between 1990 and 2014, in which gametocytes were recorded using the search strategy described in the legend of Additional file 1: Table S1. Those who had contributed studies previously to the WorldWide Antimalarial Resistance Network (WWARN) data repository were also invited to participate and asked whether they were aware of any unpublished or ongoing clinical trials involving ACTs, and these additional unpublished studies were also requested. Investigators were invited to participate in this pooled analysis if their studies included (1) uncomplicated P. falciparum malaria (alone or mixed infection with another species); (2) asexual parasite quantification at enrolment; (3) gametocyte quantification or prevalence at enrolment; (4) well described methodology for quantifying asexual parasites and gametocytes; and (5) haemoglobin (or haematocrit) estimation at enrolment.

Individual study protocols were available for all trials included, either from the publication or as a metafile submitted with the raw data. Individual patient data from eligible studies were shared, collated and standardised using a previously described methodology [10, 20]. Study reports generated from the formatted datasets were sent back to investigators for validation or clarification. All parasite data were based on microscopic observations.

\section{Statistical analysis}

Statistical analyses were carried out using STATA (Version 13.1) according to an a priori Statistical Analysis Plan [20]. Briefly, we determined: (1) prevalence of gametocytaemia at enrolment (regardless of subsequent treatment regimen); (2) risk of gametocytaemia in patients presenting with no gametocytaemia on enrolment; and (3) time to clearance of gametocytaemia in patients presenting with gametocytaemia. For the comparison of ACT regimens, the analysis was 
restricted to individuals with no recurrent asexual parasitaemia recorded during follow-up. Multivariable models with random effects were fitted to adjust for study and site heterogeneity: logistic for outcome (1) and Cox regression (with shared frailty) for outcomes (2) and (3). The effect of the following baseline covariates was examined: age, sex, log asexual parasite density, hyperparasitaemia (asexual parasitaemia $>200,000$ parasites per $\mu \mathrm{L}$ ), haemoglobin/haematocrit, anaemia (haemoglobin concentration $<10 \mathrm{~g} / \mathrm{dL}$ ), presence of/history of fever, nutritional status (based on weight-for-age z-scores in children $<5$ years of age), treatment dose of artemisinin derivative, geographic region and malaria transmission intensity [21]. Indicators of parasite clearance time included asexual parasite prevalence and log asexual parasite density on days 1, 2, 3 and the area under the curve of asexual parasite density during days $0-3$. Fractional polynomials [22] were used to define the nonlinear relationship between age, haemoglobin concentration and asexual parasite density and the risk of gametocytaemia; to maintain stability, these models were fitted to data from patients $\leq 70$ years of age, with haemoglobin between 5 and $18 \mathrm{~g} / \mathrm{dL}$ and with $500-200,000$ asexual parasites per $\mu \mathrm{L}$. Target dosing for the artemisinin components of the ACTs was defined according to WHO guidelines: $\geq 8.4 \mathrm{mg} / \mathrm{kg}$ for $\mathrm{AL}$ and $\geq 6 \mathrm{mg} / \mathrm{kg}$ for AS-AQ, AS-MQ and DP [23].

Gametocyte carriage at any time after treatment in patients with no recurrent parasitaemia, patients with recrudescent infections and patients with reinfections were compared using multilevel logistic regression models with random effects for study site and subject.

Methods to detect gametocytes by microscopy differed between trials. The sensitivity of microscopy methods was included in the analyses, by classifying studies into one of four categories, as follows: (1) studies in which slides were specifically read for gametocytes, reviewing at least 100 microscopic high power fields or against $\geq 1000$ white blood cells (WBC) (4 studies); (2) microscopists specifically instructed to record gametocytes but slides were primarily read for asexual parasites; $\geq 100$ microscopic high power fields per $\geq 1000$ WBC were read (26 studies); (3) microscopists were specifically instructed to record gametocytes; 50-99 microscopic high power fields per 500999 WBC were read (33 studies); (4) microscopists were not specifically instructed to record gametocytes or the number of examined high power fields was $<50$ or the number of WBC was $<500$ (40 studies). For 18 studies, the information on the sensitivity of the microscopy was not available.

Risk of bias within studies was assessed based on (1) study design (randomization, sequence generation, blinding); (2) methodology for gametocyte detection; and (3) the number and proportion of patients with (a) missing outcomes and (b) missing baseline covariates (age, weight, parasitaemia, temperature, haemoglobin/ haematocrit). For the final models, two sets of sensitivity analyses were performed. Firstly, a model was refitted with each study's data excluded, one at a time, and a coefficient of variation around the parameter estimates calculated. This would identify any influential studies, that is, studies with unusual results (due to variations in methodology, patient population, or other reasons) that affect the overall pooled analysis findings. Secondly, for the outcome measure time to gametocytaemia, the impact of incomplete gametocyte carriage data was investigated by refitting the final multivariable model in a subset of patients with complete weekly data for 28 days.

\section{Ethical approval}

All data included in this analysis were obtained in accordance with the laws and ethical approvals applicable to the countries in which the studies were conducted, and were obtained with the knowledge and consent of the individual to which they relate. Data were fully anonymised either before or during the process of uploading to the WWARN repository. Ethical approval to conduct individual participant data pooled analyses was granted to WWARN by the Oxford Tropical Research Ethics Committee (OXTREC-48-09).

\section{Results}

\section{Characteristics of included studies}

In total, 169 published clinical trials were identified that recorded $P$. falciparum gametocytes at enrolment or during follow-up. Investigators of 117 clinical trials $(59,458$ patients) agreed to contribute their data. In addition, nine unpublished studies (1,803 patients) were shared, one of which was published subsequently. After exclusion of duplicate studies, studies in returning travellers, multiple infection episodes and participants with protocol violations, 48,840 study participants from 121 individual clinical trials were retained (Fig. 1; full list of studies in Additional file 1: Table S1).

\section{Baseline characteristics}

The majority of participants were from Africa (34,377; 70.4 \%) or Asia $(13,546 ; 27.7 \%)$ with a minority coming from South America $(917 ; 1.9 \%$ ) (Table 1). Most studies involved treatment with an ACT $(68.3 \%$ of all participants $(33,356 / 48,840))$ with AL being the most commonly used regimen $(27.1 \% ; 13,217 / 48,840)$ (Table 2). AS-AQ was given to $17.4 \%(8488 / 48,840)$ of participants; $50.4 \%(4278 / 8488)$ of these received a fixed dose combination (FDC), others received a non-fixed dose combination $(42.9 \%$; 3637/8488) or co-blistered AS and AQ $(6.8 \% ; 573 / 8488)$. The analyses for AS-AQ were restricted to the FDC regimen (AS-AQ FDC). AS-MQ was 


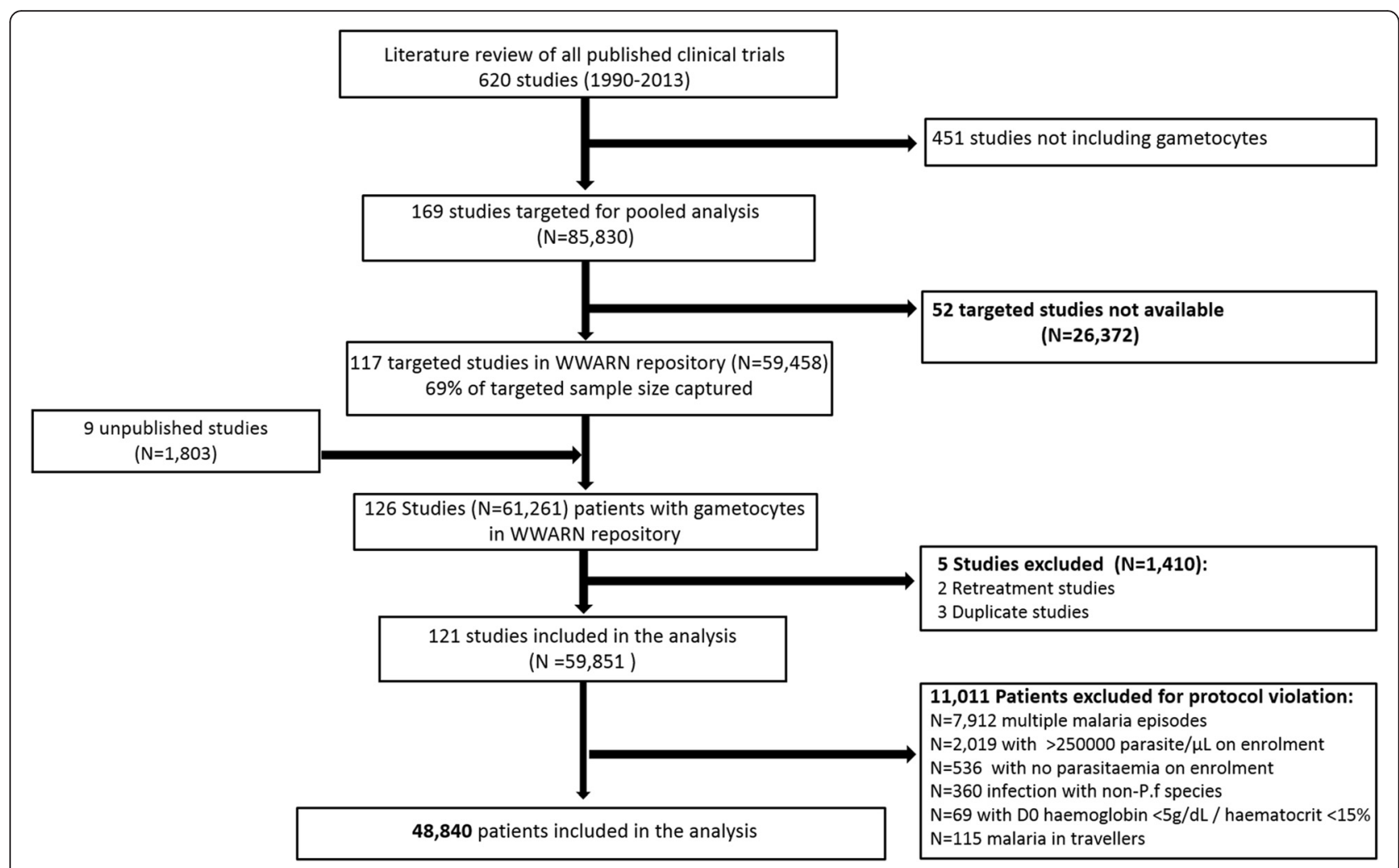

Fig. 1 Study profile

administered to $10.6 \%(5198 / 48,840)$ of participants, in most of the patients $(88.1 \% ; 4580 / 5198)$ as a loose combination. The following proportions of patients received less than the recommended dose, AL: $8.3 \%$ (1088/ 13,086); AS-AQ FDC: $0.1 \%$ (2/4262); AS-MQ: $0.8 \%$ (38/4769) DP: $23.6 \%(1488 / 6315)$.

\section{Determinants of gametocytaemia at enrolment}

Prevalence of gametocytaemia at enrolment was $12.1 \%$ (5887/48,589), and was not significantly influenced by the slide reading method. In Africa, fractional polynomial analysis indicated a gradual decline in the proportion of gametocyte-positive smears with increasing age (Fig. 2); in Asia there was an initial increase in prevalence of gametocytaemia with increasing age in the first 20 years of life, followed by a decline with increasing age thereafter. The differences between African and Asian sites in the association between age and prevalence of gametocytaemia remained apparent when the analysis was restricted to studies with the highest sensitivity of gametocyte detection ( $\geq 100$ high power fields or $\geq 1,000$ WBC examined specifically for gametocytes) and when restricted to children below 5 years of age (Additional file 2: Figure S1). Prevalence of gametocytaemia at enrolment was negatively associated with haemoglobin concentration in all three continents (Table 3; Fig. 2).
In Asia, there was a gradual decline in prevalence of gametocytaemia with increasing asexual parasite density across the entire range of asexual parasite densities that were observed (Fig. 2). In Africa, when asexual parasite density exceeded 10,000 parasites $/ \mu \mathrm{L}$, there was a gradual decline in prevalence of gametocytaemia with increasing asexual parasite density. At lower parasite densities the uncertainty around estimates was larger and the association between prevalence of gametocytaemia and the logarithm of asexual parasite density was non-linear (Fig. 2). These differences between African and Asian sites remained apparent when the analysis was restricted to studies with the highest sensitivity of gametocyte detection (Additional file 2: Figure S1).

In all regions, individuals presenting with fever (axillary temperature $>37.5{ }^{\circ} \mathrm{C}$ or reporting of febrile symptoms) were less likely to present with gametocytaemia and this remained significant after adjusting for covariates in both African (adjusted odds ratio (AOR), 0.63; $95 \% \mathrm{CI}, 0.58-0.69 ; P<0.001$ ) and Asian (AOR, $0.81 ; 95 \% \mathrm{CI}, 0.69-0.95 ; P=0.011$ ) patients. Male gender was a predictor of prevalence of gametocytaemia at enrolment in studies in Asia (AOR, 1.25; $95 \% \mathrm{CI}$, 1.07-1.46; $P=0.004$ ) and South America (AOR, 2.14; $95 \%$ CI, 1.33-3.45; $P=0.002$ ) but not Africa (Table 3). Children under 5 years of age who were malnourished 
Table 1 Demographic and baseline characteristics

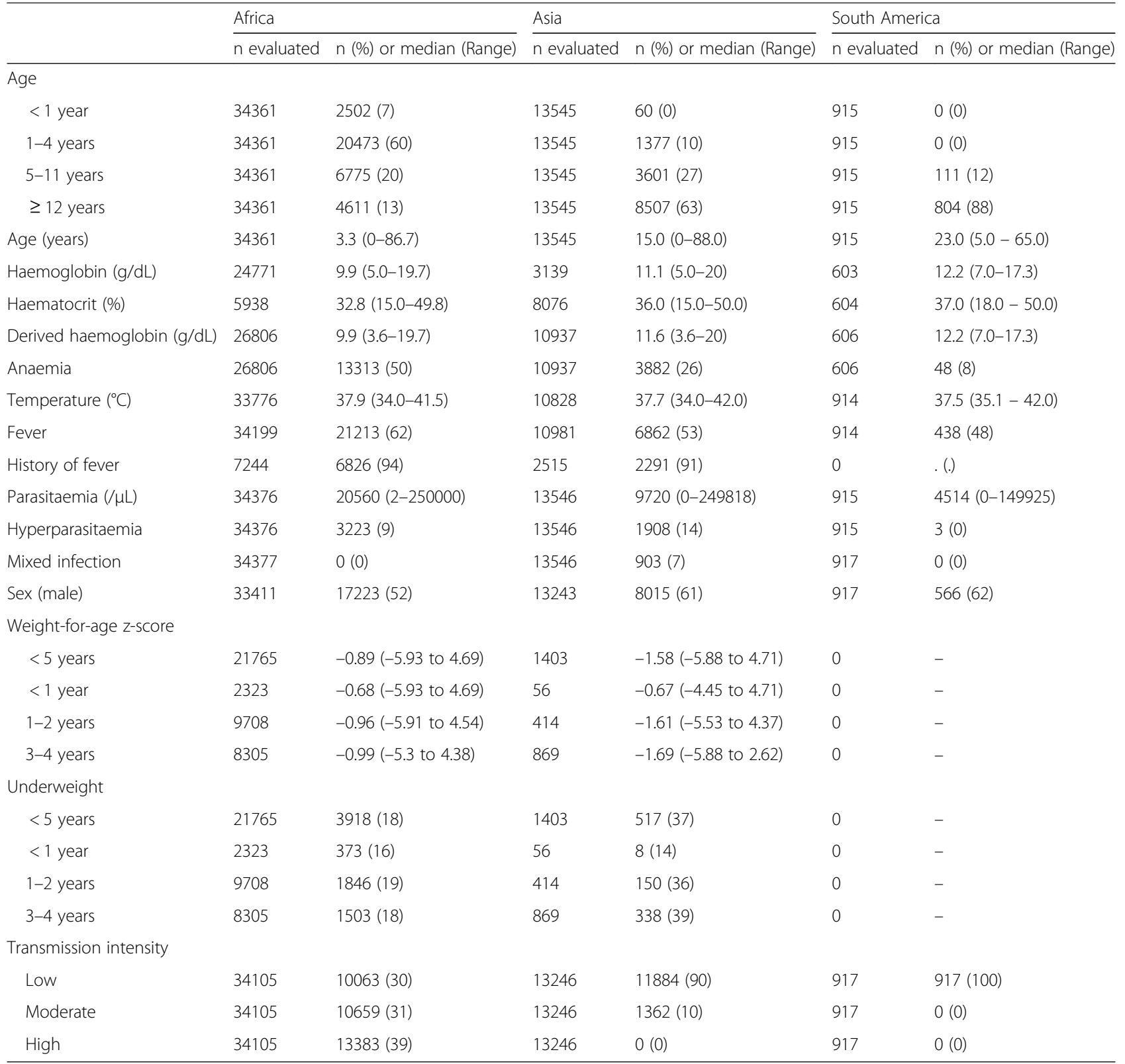

Derived haemoglobin, conversion from haematocrit: haemoglobin = (haematocrit-5.62)/2.60 [40]; Anaemia, haemoglobin $<10 \mathrm{~g} / \mathrm{dL}$; Fever, temperature $>37.5^{\circ} \mathrm{C}$; Hyperparasitaemia, parasitaemia > 100,000 parasites per $\mu \mathrm{L}$; Weight-for-age $z$-score, calculated using "igrowup" package developed by WHO [41] in children $<5$ years of age; Underweight, weight-for-age z-scores $<-2$

(weight-for-age $\mathrm{z}$-scores $<-2$ ) had a higher prevalence of gametocytaemia at enrolment compared to wellnourished children in Africa (OR, 1.23; $95 \% \mathrm{CI}$, $1.11-1.37 ; P<0.001)$ and in Asia $(\mathrm{OR}, 1.40 ; 95 \% \mathrm{CI}$, $1.03-1.92 ; P=0.032)$ but this was not significant in the multivariable analysis (Additional file 3: Table S2).

\section{Gametocytaemia after artemisinin combination therapy No gametocytaemia at enrolment}

Amongst the 18,388 individuals presenting without patent gametocytaemia by microscopy who were treated with an ACT, the Kaplan-Meier estimate of risk of appearance of gametocytaemia within 28 days was $1.9 \%$ (95\% CI, 1.7-2.1) (Fig. 3a). This proportion was similar in African and Asian studies. After controlling for confounding factors, the risk of appearance of gametocytaemia correlated negatively with age, haemoglobin concentration, fever and asexual parasite density at enrolment (Table 4). Appearance of gametocytaemia was lowest after AS-MQ or AL treatment and significantly higher after DP (adjusted hazard ratio (AHR), 2.03; $95 \% \mathrm{CI}, 1.24-3.32 ; P=0.005$ compared to $\mathrm{AL}$ ) 
Table 2 Overview of treatment, artemisinin combination treatment dosing and formulation

\begin{tabular}{|c|c|c|c|c|c|c|}
\hline & \multicolumn{2}{|l|}{ Treatment } & \multicolumn{4}{|l|}{ Dosing } \\
\hline & $\mathrm{n}$ evaluated & N (\%) & $\mathrm{n}$ evaluated & $\begin{array}{l}\text { Partner drug dose } \\
\text { median (Range) }\end{array}$ & $\begin{array}{l}\text { Artemisinin derivative dose } \\
\text { median (Range) }\end{array}$ & Underdosed $\mathrm{n}(\%)$ \\
\hline$\overline{A L}$ & 48840 & $13217(27 \%)$ & 13086 & $68.6(8.9-144.0)$ & $11.4(1.5-24.0)$ & $1008(8.3 \%)$ \\
\hline AS-AQ & 48840 & $8488(17 \%)$ & 8395 & $31.9(10.0-91.8)$ & $12.4(4.0-52.6)$ & \\
\hline \multicolumn{7}{|l|}{ AS-AQ formulation: } \\
\hline Co-blistered nFDC & 8488 & $573(7 \%)$ & 573 & $37.4(14.8-91.8)$ & $13.5(4.8-30.0)$ & \\
\hline FDC & 8488 & $4278(50 \%)$ & 4262 & $32.4(14.5-81.0)$ & $12.0(5.4-30.0)$ & $2(0.1 \%)$ \\
\hline $\mathrm{nFDC}$ & 8488 & 3637 (43\%) & 3560 & $30.1(10.0-60.0)$ & $12.5(4.0-52.6)$ & \\
\hline AS-MQ & 48840 & $5198(11 \%)$ & 4535 & $25.0(4.2-85.0)$ & $12.0(2.3-62.1)$ & 38 (0.8 \%) \\
\hline DP & 48840 & $6453(13 \%)$ & 6315 & $53.3(14.5-182.9)$ & $6.7(1.8-22.9)$ & $1488(23.6 \%)$ \\
\hline Other, including non-ACT & 48840 & 15484 (32 \%) & & & & \\
\hline
\end{tabular}

AL, Artemether-Lumefantrine; AS-AQ, Artesunate-Amodiaquine; AS-MQ, Artesunate-Mefloquine; DP, Dihydroartemisinin-piperaquine; $\mathrm{nFDC}$, Non-fixed dose combination, FDC, Fixed dose combination; Underdosed defined as $\leq 8.4 \mathrm{mg} / \mathrm{kg}$ artemether dose in $\mathrm{AL},<6 \mathrm{mg} / \mathrm{kg}$ dose of artesunate or DHA in other regimens [19]

and AS-AQ (AHR, 4.01; $95 \%$ CI, 2.40-6.72; $P<0.001$ compared to AL) (Fig. 3a, Table 4). A dose of the artemisinin component $<8 \mathrm{mg} / \mathrm{kg}$ was associated with an increased chance of appearance of gametocytaemia after treatment with DP (AHR, 2.78; 95 \% CI, 1.18-
6.55; $P=0.020)$ but not after treatment with any of the other ACTs (Additional file 4: Table S3). No association was observed for a dose of the artemisinin component $<6 \mathrm{mg} / \mathrm{kg}$ either for all treatment combined or for DP alone.

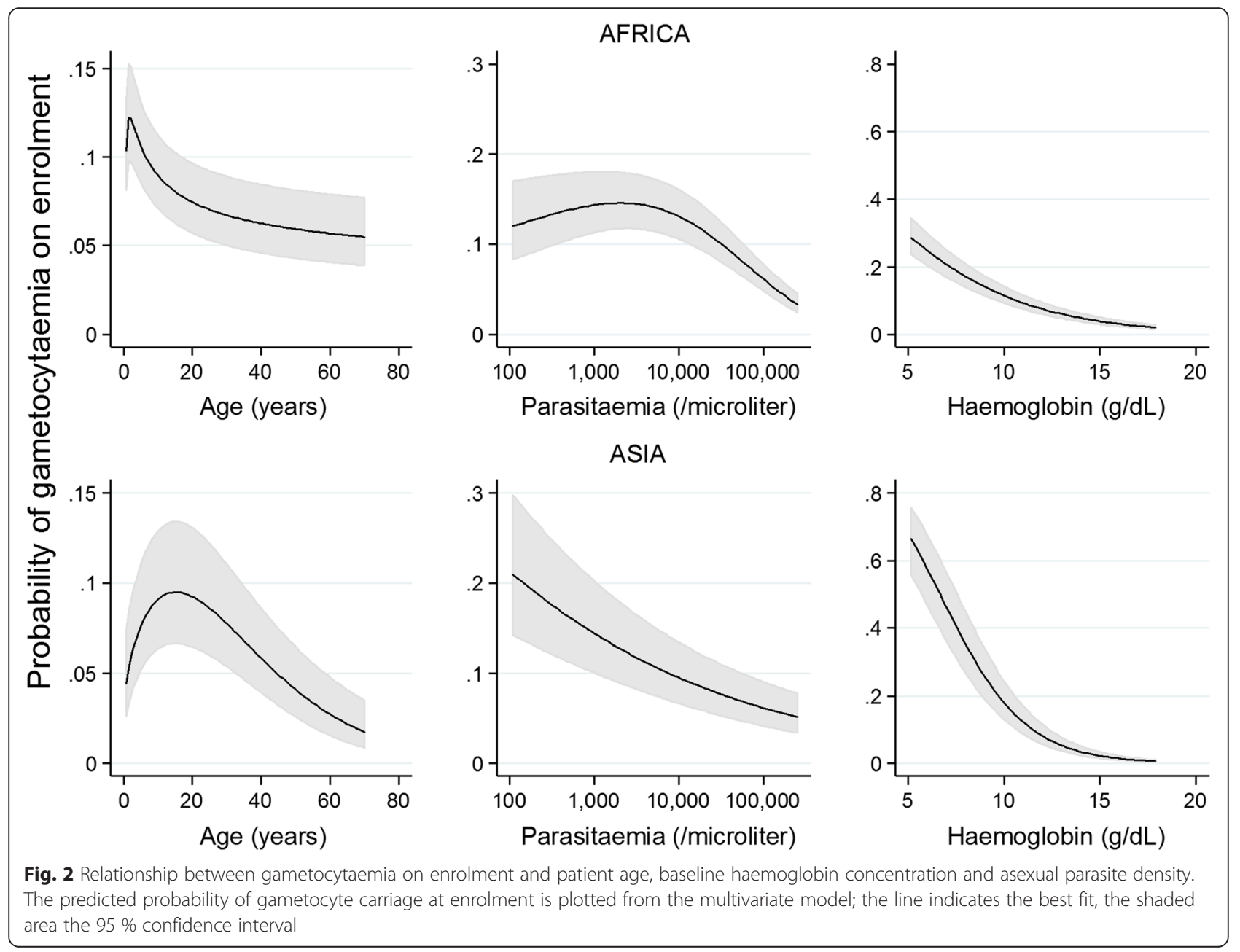


Table 3 Risk factors for gametocyte prevalence at enrolment

\begin{tabular}{|c|c|c|c|c|c|c|c|c|c|}
\hline \multirow[b]{2}{*}{ Parameter } & \multicolumn{3}{|l|}{ Africa } & \multicolumn{3}{|l|}{ Asia } & \multicolumn{3}{|l|}{ South America } \\
\hline & Nobs/Npos (\%) & OR $(95 \%$ Cl) & $P$ value & Nobs/Npos (\%) & OR $(95 \% \mathrm{Cl})$ & $P$ value & Nobs/Npos (\%) & OR $(95 \%$ Cl) & $P$ value \\
\hline \multicolumn{10}{|l|}{ Univariable model } \\
\hline \multicolumn{10}{|l|}{ Age } \\
\hline$<1$ year & 2492/403 (16.2) & $2.506(1.978-3.174)$ & $<0.001$ & $60 / 12(20.0)$ & $2.240(1.124-4.460)$ & 0.022 & $0 / 0$ & & \\
\hline $1-4$ years & 20419/2799 (13.7) & $2.558(2.082-3.144)$ & $<0.001$ & 1374/297 (21.6) & $2.095(1.777-2.469)$ & $<0.001$ & $0 / 0$ & & \\
\hline $5-11$ years & $6715 / 495(7.4)$ & $1.523(1.245-1.864)$ & $<0.001$ & $3587 / 563(15.7)$ & $1.535(1.354-1.740)$ & $<0.001$ & $111 / 19(17.1)$ & $0.834(0.485-1.432)$ & 0.510 \\
\hline $12+$ years & $4571 / 179(3.9)$ & - & & $8438 / 977(11.6)$ & - & & 803/139 (17.3) & - & \\
\hline Age (years) & $34197 / 3876(11.3)$ & $0.961(0.952-0.971)$ & $<0.001$ & $13459 / 1849(13.7)$ & $0.975(0.971-0.980)$ & $<0.001$ & $914 / 158(17.3)$ & $0.995(0.983-1.008)$ & 0.453 \\
\hline Derived haemoglobin ( $\mathrm{g} / \mathrm{dL})$ & 26693/3394 (12.7) & $0.785(0.767-0.803)$ & $<0.001$ & 10854/1433 (13.2) & $0.664(0.645-0.683)$ & $<0.001$ & 606/120 (19.8) & $0.600(0.523-0.688)$ & $<0.001$ \\
\hline \multicolumn{10}{|l|}{ Anaemia } \\
\hline Yes & 13441/2322 (17.3) & $2.062(1.888-2.253)$ & $<0.001$ & $2871 / 795(27.7)$ & $4.846(4.258-5.516)$ & $<0.001$ & $48 / 22(45.8)$ & $3.972(2.162-7.297)$ & $<0.001$ \\
\hline No & 13252/1072 (8.1) & Reference & & 7983/638 (8.0) & Reference & & 558/98 (17.6) & Reference & \\
\hline \multicolumn{10}{|l|}{ Fever } \\
\hline Yes & 21115/1910 (9.1) & $0.583(0.538-0.631)$ & $<0.001$ & $5816 / 566(9.7)$ & $0.738(0.649-0.839)$ & $<0.001$ & 438/54 (12.3) & $0.529(0.367-0.762)$ & 0.001 \\
\hline No & 12925/1950 (15.1) & Reference & & $5107 / 730$ (14.3) & Reference & & 476/105 (22.1) & Reference & \\
\hline \multicolumn{10}{|l|}{ Sex } \\
\hline Female & 16119/1782 (11.1) & $0.976(0.908-1.048)$ & 0.501 & $5205 / 754(14.5)$ & $1.083(0.973-1.206)$ & 0.145 & 350/52 (14.9) & $0.756(0.525-1.089)$ & 0.133 \\
\hline Male & 17128/1963 (11.5) & Reference & & 7952/1085 (13.6) & Reference & & 566/107 (18.9) & Reference & \\
\hline 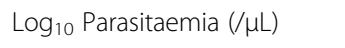 & $34212 / 3879$ (11.3) & $0.590(0.554-0.629)$ & $<0.001$ & 13442/1833 (13.6) & $0.726(0.677-0.779)$ & $<0.001$ & 914/158 (17.3) & $0.333(0.212-0.524)$ & $<0.001$ \\
\hline \multicolumn{10}{|l|}{ Hyperparasitaemia } \\
\hline Yes & $3217 / 160(5.0)$ & $0.389(0.328-0.460)$ & $<0.001$ & 1892/289 (15.3) & $0.441(0.338-0.575)$ & $<0.001$ & $0 / 0$ & & \\
\hline No & $30995 / 3719$ (12.0) & Reference & & 12568/1743 (13.9) & Reference & & $912 / 158(17.3)$ & & \\
\hline \multicolumn{10}{|l|}{ Mixed infection } \\
\hline Yes & & & & 892/106 (11.9) & $1.112(0.880-1.404)$ & 0.374 & & & \\
\hline No & & & & 13496/1817 (13.5) & Reference & & & & \\
\hline Weight-for-age z-score & 21701/2996 (13.8) & $0.932(0.901-0.966)$ & $<0.001$ & $1403 / 305(21.7)$ & $0.815(0.723-0.919)$ & 0.001 & $0 / 0$ & & \\
\hline \multicolumn{10}{|l|}{ Underweight } \\
\hline Yes & $3904 / 651(16.7)$ & $1.234(1.113-1.368)$ & $<0.001$ & $517 / 144(27.9)$ & 1.404 (1.029-1.915) & 0.032 & $0 / 0$ & & \\
\hline No & 17797/2345 (13.2) & Reference & & 886/161 (18.2) & Reference & & $0 / 0$ & & \\
\hline \multicolumn{10}{|l|}{ TIA } \\
\hline Low & $9995 / 802(8.0)$ & $0.990(0.674-1.454)$ & 0.959 & 11799/1383 (11.7) & $0.251(0.074-0.850)$ & 0.026 & $871 / 147$ (16.9) & & \\
\hline Moderate & 10575/1069 (10.1) & $1.074(0.801-1.440)$ & 0.631 & $1361 / 438(32.2)$ & Reference & & $0 / 0$ & & \\
\hline High & 13371/2000 (15.0) & Reference & & $0 / 0$ & & & $0 / 0$ & & \\
\hline
\end{tabular}


Table 3 Risk factors for gametocyte prevalence at enrolment (Continued)

\begin{tabular}{|c|c|c|c|c|c|c|c|c|c|}
\hline Multivariable model & 26669 / 3389 (12.7) & & & 8919 / $929(10.4)$ & & & $605 / 120$ (19.8) & & \\
\hline Age (years) & & $0.984(0.974-0.994)$ & 0.001 & & $0.988(0.982-0.994)$ & $<0.001$ & & & \\
\hline Derived haemoglobin ( $\mathrm{g} / \mathrm{dL}$ ) & & $0.788(0.770-0.807)$ & $<0.001$ & & $0.672(0.648-0.697)$ & $<0.001$ & & $0.581(0.502-0.672)$ & $<0.001$ \\
\hline 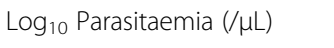 & & $0.617(0.575-0.662)$ & $<0.001$ & & $0.735(0.669-0.807)$ & $<0.001$ & & $0.330(0.184-0.592)$ & $<0.001$ \\
\hline Fever & & $0.633(0.579-0.691)$ & $<0.001$ & & $0.811(0.689-0.954)$ & 0.011 & & & \\
\hline $\operatorname{Sex}(M)$ & & & & & $1.252(1.073-1.462)$ & 0.004 & & $2.144(1.331-3.454)$ & 0.002 \\
\hline
\end{tabular}

Logistic univariable and multivariable mixed effects analysis by region with presence of gametocytaemia at enrolment as dependent variable. Nobs, number of observations; Npos, number of positive observations; Weight-for-age $z$-score, calculated using "igrowup" package developed by WHO [41] in children $<5$ years of age; Underweight, weight-for-age $z$-scores $<-2$; TIA, Transmission intensity areas; Derived haemoglobin, conversion from haematocrit: haemoglobin $=$ (haematocrit-5.62)/2.60 [40]; Anaemia, haemoglobin $<10 \mathrm{~g} / \mathrm{dL}$; Fever, temperature $>37.5^{\circ} \mathrm{C}$; Hyperparasitaemia, parasitaemia $>100,000$ parasites per $\mu \mathrm{L}$ 

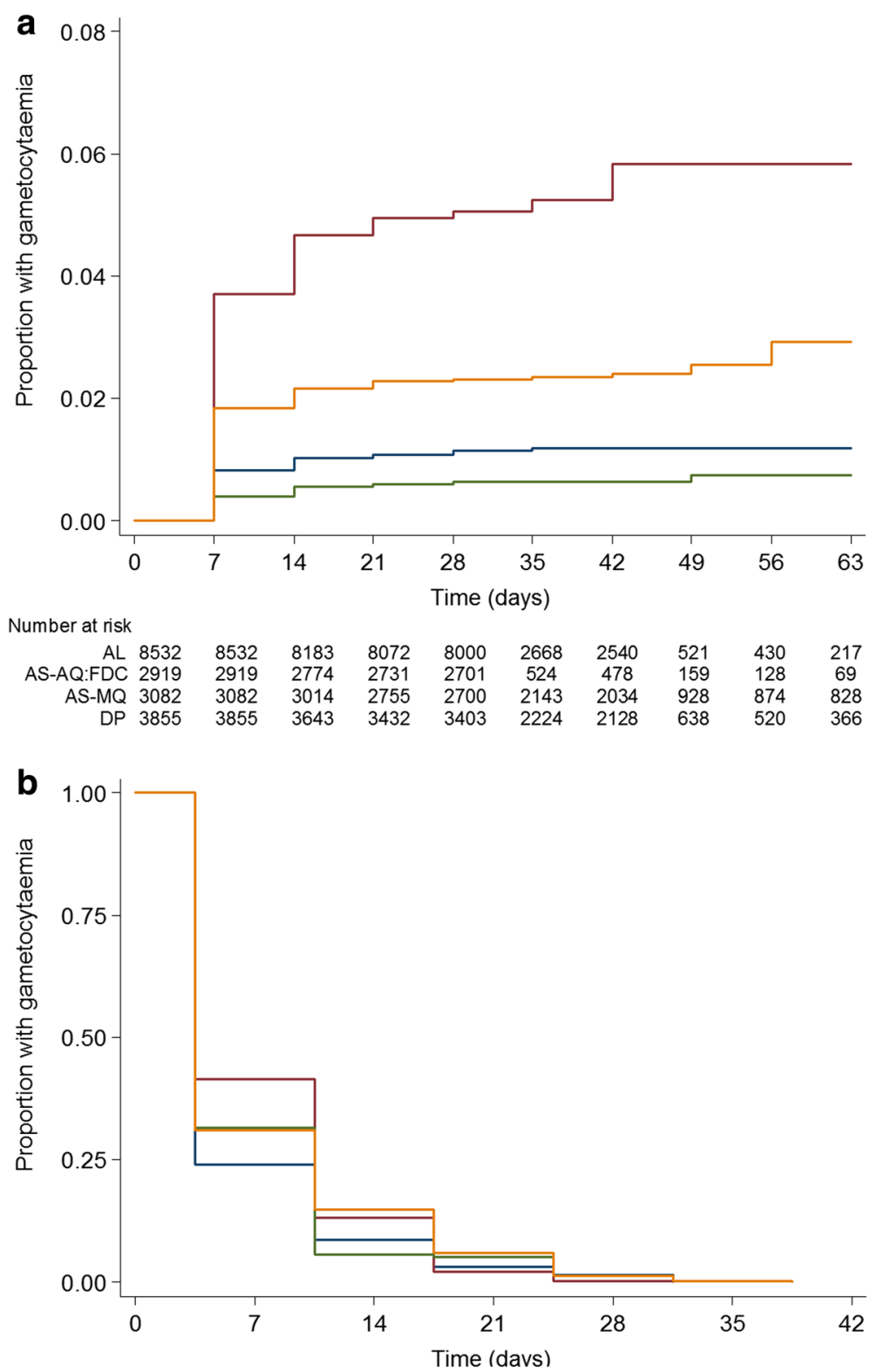

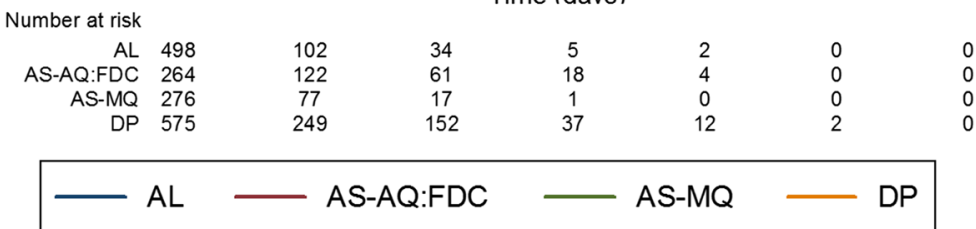

Fig. 3 Gametocyte carriage by artemisinin-combination therapy. a Development of gametocytaemia after treatment with artemether-lumefantrine $(\mathrm{AL})$, artesunate-amodiaquine fixed-dose combination (AS-AQ-FDC), artesunate-mefloquine (AS-MQ) or dihydroartemisinin-piperaquine (DP); evaluated in patients with no gametocytes on enrolment. b Gametocyte clearance, adjusted for initial gametocyte count, evaluated in patients with gametocytes on enrolment. Only patients with no recurrent infection recorded were included

\section{Gametocytaemia at enrolment}

A total of 2433 patients treated with an ACT were gametocytaemic at enrolment and had no recurrent infection. Overall, 57.4 \% (95\% CI, 55.4-59.4) of these patients cleared gametocytaemia by day 7, 78.4 \% (95\% CI, 76.5-80.2) by day 14 and $88.2 \%$ (95 \% CI, 86.6$89.6)$ by day 21 . The only independent determinants of gametocyte clearance were initial gametocyte density 
Table 4 Factors associated with the development of gametocytaemia after enrolment in individuals without microscopically detected gametocytes before treatment with artemisinin combination therapy

\begin{tabular}{|c|c|c|c|c|c|}
\hline Parameter & Nobs & Npos & per & Hazard ratio $(95 \%$ Cl) & $P$ value \\
\hline \multicolumn{6}{|l|}{ Univariable model } \\
\hline \multicolumn{6}{|l|}{$\mathrm{ACT}^{\mathrm{a}}$} \\
\hline AS-MQ & 3082 & 20 & 0.6 & $0.763(0.392-1.484)$ & 0.425 \\
\hline DP & 3855 & 93 & 2.4 & $2.746(1.773-4.253)$ & $<0.001$ \\
\hline AS-AQ: FDC & 2919 & 151 & 5.2 & $4.094(2.540-6.600)$ & $<0.001$ \\
\hline $\mathrm{AL}$ & 8532 & 97 & 1.1 & Reference & \\
\hline \multicolumn{6}{|l|}{$\mathrm{Age}^{\mathrm{a}}$} \\
\hline$<1$ year & 776 & 23 & 3.0 & $2.435(1.268-4.676)$ & 0.008 \\
\hline $1-4$ years & 7772 & 236 & 3.0 & $2.780(1.698-4.552)$ & $<0.001$ \\
\hline $5-11$ years & 4102 & 58 & 1.4 & $1.928(1.225-3.035)$ & 0.005 \\
\hline $12+$ years & 5735 & 44 & 0.8 & Reference & \\
\hline Age (years) & 18385 & 361 & 2.0 & $0.965(0.946-0.984)$ & $<0.001$ \\
\hline Derived haemoglobin $(\mathrm{g} / \mathrm{dL})$ & 14357 & 295 & 2.1 & $0.809(0.758-0.862)$ & $<0.001$ \\
\hline \multicolumn{6}{|l|}{ Anaemia } \\
\hline Yes & 5505 & 183 & 3.3 & $1.824(1.402-2.373)$ & $<0.001$ \\
\hline No & 8852 & 112 & 1.3 & Reference & \\
\hline \multicolumn{6}{|l|}{ Fever } \\
\hline Yes & 10569 & 173 & 1.6 & $0.594(0.470-0.749)$ & $<0.001$ \\
\hline No & 7244 & 173 & 2.4 & Reference & \\
\hline \multicolumn{6}{|l|}{ Sex } \\
\hline Female & 8427 & 160 & 1.9 & $0.931(0.754-1.149)$ & 0.505 \\
\hline Male & 9658 & 196 & 2.0 & Reference & \\
\hline \multicolumn{6}{|l|}{ Hyperparasitaemia } \\
\hline Yes & 1543 & 19 & 1.2 & $0.615(0.384-0.984)$ & 0.043 \\
\hline No & 16845 & 342 & 2.0 & Reference & \\
\hline $\log _{10}$ parasitaemia $(/ \mu \mathrm{L})$ & 18388 & 361 & 2.0 & $0.731(0.619-0.864)$ & $<0.001$ \\
\hline \multicolumn{6}{|l|}{ Weight-for-age score } \\
\hline Underweight $^{\mathrm{b}}$ & 8341 & 257 & 3.1 & $0.825(0.744-0.915)$ & $<0.001$ \\
\hline Yes & 1418 & 80 & 5.6 & $1.768(1.343-2.326)$ & $<0.001$ \\
\hline No & 6923 & 177 & 2.6 & Reference & \\
\hline \multicolumn{6}{|l|}{ Region } \\
\hline Asia & 3895 & 55 & 1.4 & $1.078(0.356-3.263)$ & 0.894 \\
\hline South America & 615 & 8 & 1.3 & $0.482(0.035-6.564)$ & 0.584 \\
\hline Africa & 13878 & 298 & 2.1 & Reference & \\
\hline \multicolumn{6}{|l|}{$T I A^{a}$} \\
\hline Low & 8406 & 64 & 0.8 & $0.371(0.159-0.866)$ & 0.022 \\
\hline Moderate & 5120 & 129 & 2.5 & $0.746(0.426-1.306)$ & 0.305 \\
\hline High & 4449 & 161 & 3.6 & Reference & \\
\hline Multivariable model & 14051 & 291 & 2.1 & & \\
\hline \multicolumn{6}{|l|}{ ACT: } \\
\hline AS-MQ & & & & $0.566(0.225-1.420)$ & 0.225 \\
\hline DP & & & & $2.029(1.240-3.317)$ & 0.005 \\
\hline AS-AQ: FDC & & & & 4.014 (2.398-6.719) & $<0.001$ \\
\hline $\mathrm{AL}$ & & & & Reference & \\
\hline
\end{tabular}


Table 4 Factors associated with the development of gametocytaemia after enrolment in individuals without microscopically detected gametocytes before treatment with artemisinin combination therapy (Continued)

\begin{tabular}{lll}
\hline Age & & \\
$<1$ year & $1.707(0.778-3.747)$ & 0.269 \\
$1-4$ years & $2.303(1.208-4.392)$ & 0.011 \\
$5-11$ years & $1.418(0.795-2.527)$ & 0.237 \\
$12+$ years & Reference & \\
Derived haemoglobin $(\mathrm{g} / \mathrm{dL})$ & $0.828(0.774-0.886)$ & $<0.001$ \\
Fever & $0.653(0.503-0.848)$ & 0.001 \\
Log $_{10}$ parasitaemia $(/ \mu \mathrm{L})$ & $0.757(0.624-0.917)$ & 0.004 \\
\hline
\end{tabular}

Cox regression mixed effects model for time to gametocytaemia

Nobs, Number of observations; Npos, Number of positive observations; Weight-for-age z-score, calculated using "igrowup" package developed by WHO [41]

in children < 5 years of age; Underweight, weight-for-age $z$-scores <-2; TIA, Transmission intensity areas. ${ }^{a}$ Proportional hazards assumption not satisfied;

b In multivariable analysis: $\mathrm{HR}, 1.51 ; 95 \% \mathrm{Cl}, 1.13-2.02 ; P=0.005$, after adjusting for covariates in the main model

(AHR, 0.87; $95 \% \mathrm{CI}, 0.83-0.91 ; P<0.001$ for $\log$ increase in gametocyte density) and the type of ACT given (Additional file 5: Table S4, Fig. 3b). Compared to AL, gametocytaemia clearance was significantly faster with AS-MQ (AHR, 1.26; $95 \% \mathrm{CI}, 1.00-1.60 ; P=0.054$ ) and slower with DP (AHR, 0.74; $95 \% \mathrm{CI}, 0.63-0.88$; $P=0.001$ ) (Fig. 3b). For the AS-AQ FDC, the rate of gametocytaemia clearance was significantly slower compared to that of AS-MQ (HR, 0.64; $95 \%$ CI, 0.48-0.85; $P=0.002$ ), and non-significantly slower compared to AL (HR, 0.80; $95 \% \mathrm{CI}, 0.63-1.02 ; P=0.072)$. The overall observed proportion of patients who cleared gametocytes by day 7 was $64.4 \%$ for $\mathrm{AL}, 61.7 \%$ for AS-MQ, $52.3 \%$ for DP, and $47.8 \%$ for AS-AQ, while by day 14 gametocytes were cleared by $85.7 \%, 90.2 \%$, $70.3 \%$, and $72.1 \%$ of patients, respectively.

\section{Gametocytaemia in relation to asexual parasite clearance time and treatment response}

Asexual parasite clearance was rapid for all treatments with $8.8 \%, 9.1 \%, 6.4 \%$, and $7.8 \%$ of patients having residual asexual parasites after 2 days treatment with $\mathrm{AL}$, AS-MQ, AS-AQ-FDC, and DP, respectively. On day 3, these figures were $0.8 \%, 1.3 \%, 0.4 \%$, and $0.7 \%$. Residual asexual parasite prevalence on day 1,2 or 3 was not associated with gametocytaemia clearance or the appearance of gametocytaemia in univariable or multivariable analysis. Individuals who experienced PCRconfirmed treatment failure by day 28 were more likely to be gametocytaemic on any day during follow-up (AOR, 2.12; $95 \% \mathrm{CI}, 1.08-4.34 ; P=0.025$ ) and develop gametocytaemia after day 7 (AOR, 9.05; $95 \%$ CI, 3.7421.91; $P<0.001)$ compared to patients with no recorded recurrence and at least 28 days follow-up. Similarly, the increased risk of gametocytaemia on any day during follow-up (AOR, 1.95; $95 \% \mathrm{CI}, 1.37-2.77 ; P<0.001$ ) and of developing gametocytaemia after day 7 (AOR, 3.03; $95 \%$ CI, 1.66-5.54; $P<0.001$ ) was observed in individuals with reinfection (Fig. 4a). This association was not explained by differences in artemisinin dosing. Gametocytaemia clearance in individuals with gametocytaemia prior to treatment was not associated with treatment outcome (Fig. 4b).

\section{Assessment of potential bias}

Attrition bias of the included studies is presented in Additional file 6: Table S5. Although many studies were not blinded, the blinding of the independent outcome laboratory assessments (i.e. microscopy readings to measure gametocytaemia and PCR classification of treatment outcome are performed by laboratory staff not directly involved in the study), minimize the risk of bias in outcome assignment. We consider publication bias unlikely since gametocytaemia measurements were a primary outcome in only 2 (out of 121) publications and gametocytaemia results are unlikely to have influenced the decision to publish. Sensitivity analyses showed that exclusion of any of the studies did not change the main conclusions of the analysis (Additional file 7: Table S6). Results for time to gametocytaemia were also confirmed for all covariates except for age when analysis was restricted to individuals with complete weekly data on gametocytaemia (Additional file 8: Table S7 and Additional file 9: Figure S2). The fact that the effect of age was lost may be due to a considerable loss of observations in this sub-analysis that differed by age groups: $12 \%, 15 \%, 33 \%$, and $32 \%$ of patients in groups $<1$ year, $1-4$ years, $5-11$ years, and $\geq 12$ years of age were not included in the sub-analysis.

\section{Discussion}

We analysed data from nearly 50,000 patients from trials that included measures of gametocytaemia by blood smears. The prevalence of gametocytaemia before and after treatment was greatest in young patients, and those with lower asexual parasite density, anaemia and absence 

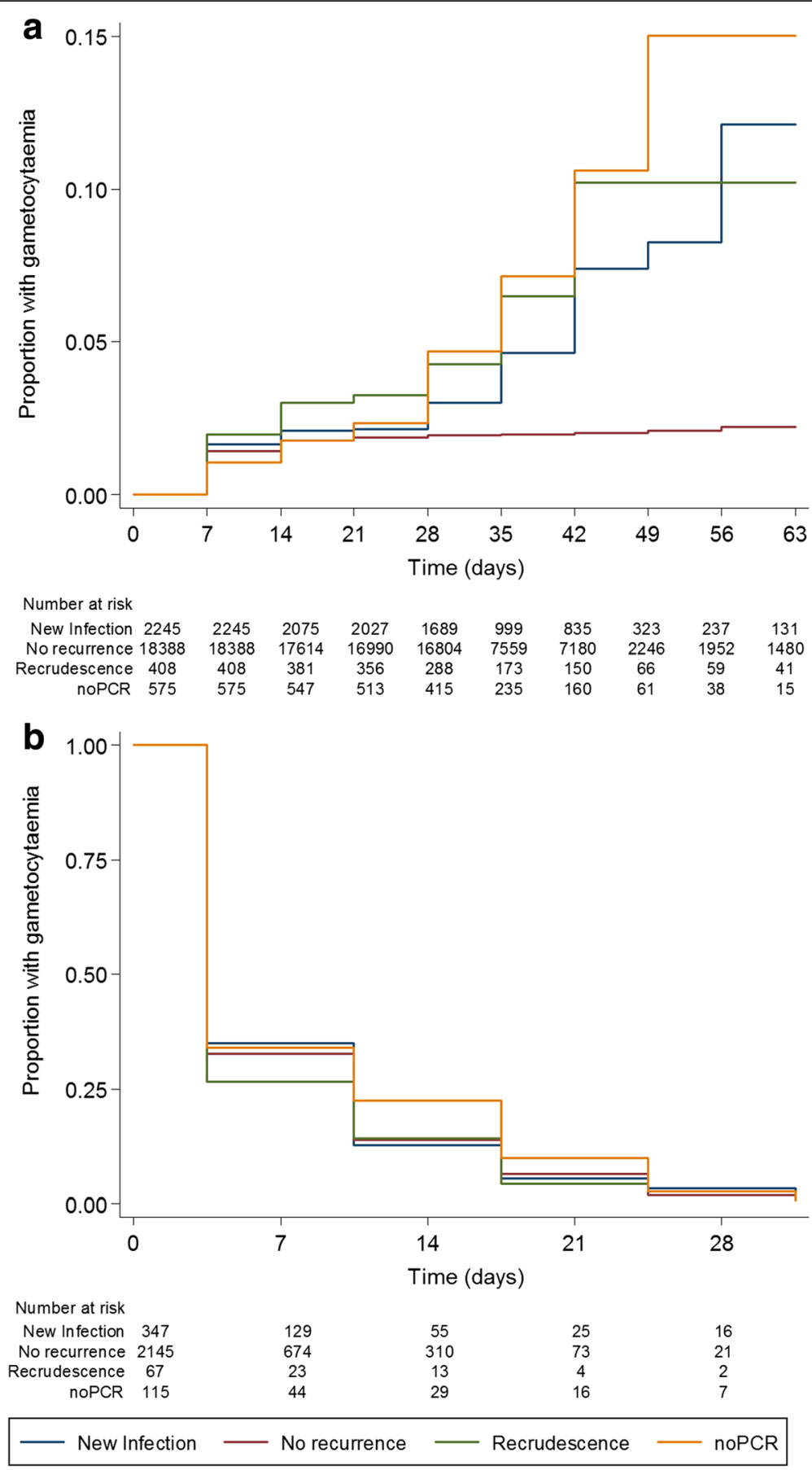

Fig. 4 Gametocyte carriage by treatment outcome. a Development of gametocytaemia after treatment; evaluated in patients with no gametocytes on enrolment. b Clearance of gametocytaemia, adjusted for initial gametocyte count, evaluated in patients with gametocytaemia on enrolment

of fever. After treatment with an ACT, the appearance and clearance of gametocytaemia was determined by the type of ACT with AL and AS-MQ being most efficacious in preventing post-treatment gametocyte carriage.

Gametocytaemia is essential for onward transmission of malaria infections to mosquitoes. Understanding factors that influence gametocytaemia prior to treatment and the gametocytocidal properties of antimalarial drugs is of great relevance for interventions that aim to reduce malaria transmission. Mature $P$. falciparum gametocytes first appear in the human bloodstream 7 to 15 days after the initial wave of their asexual parasite progenitors. This long maturation process and the impacts of human and parasite factors associated with gametocyte 
production [5] result in considerable variation in the proportion of malaria patients harbouring gametocytes upon presentation with clinical illness. We observed that the same host characteristics influenced gametocytaemia before and after treatment. The prevalence of gametocytaemia was higher in patients with anaemia and without concurrent fever [4, 24]. Reduced haemoglobin concentrations are often a consequence of prolonged duration of infections or recurrent malaria episodes [25, 26], both of which have been associated with increased gametocyte production [4]. Anaemia may also be an independent predictor of gametocytaemia [4, 27] since low haemoglobin concentrations and reticulocytosis directly stimulate gametocyte production $[28,29]$. The association between asexual parasite density at enrolment and gametocytaemia was different in Asian and African settings. In Asian studies, the prevalence of gametocytaemia showed a gradual negative association with asexual parasite density [4], whilst in Africa, this negative association was only apparent at asexual parasite densities above 5,000 parasites $/ \mu \mathrm{L}$. These setting-dependent patterns may explain previous inconsistent reports on the association between asexual parasite densities and gametocytaemia $[4,27,30,31]$. These three predictors of gametocytaemia (anaemia, lower asexual parasite density and absence of fever) may all reflect chronic infections that, because of their longer duration, may be more likely to present with gametocytaemia. Host immunity and the likelihood of super-infections vary significantly with transmission intensity and both influence asexual parasite densities and gametocyte dynamics. Age is a useful surrogate of immunity. In African studies, there was a gradual decrease in the prevalence of gametocytaemia with increasing age, while in Asia, the prevalence of gametocytaemia increased until approximately 20 years of age followed by a general decline thereafter. Further studies are needed to determine whether this pattern is explained by host-factors or by age or occupationassociated malaria exposure in Asian settings.

Patients presenting with gametocytaemia cleared their gametocytaemia rapidly following ACT, with $57 \%$ of patients being gametocyte-free by day 7 and $88 \%$ by day 21 . The rate of gametocytaemia clearance varied significantly with the ACT regimen. Differential effects of ACT on post-treatment gametocytaemia have been reported previously, but with contradicting results [32-34]. Our large meta-analysis revealed that both the appearance and duration of gametocytaemia were 2-fold and $25 \%$ lower, respectively, in AL- compared to DPtreated patients. In individuals treated with DP, a lower treatment dose was associated with an increased appearance of gametocytaemia after treatment. We previously demonstrated that treatment failure is also associated with DP dosing [10] and the World Health Organization recently increased the dose recommendation for DP to ensure a minimum of $7.5 \mathrm{mg} / \mathrm{kg}$ total dose of dihydroartemisinin in children $<25 \mathrm{~kg}$ [35]. The appearance of gametocytaemia after AS-AQ FDC was markedly more prevalent than after either AL or AS-MQ. Furthermore, gametocytaemia clearance was slower after AS-AQ FDC compared to AS-MQ. This striking difference of AS-AQ FDC compared to AL and AS-MQ could not be explained by differences in total artemisinin dosing or treatment outcome. These differential effects of ACTs may relate to the frequency of artemisinin dosing or to the activity of the non-artemisinin partner drug. In vitro drug screening assays indicate similar activity of lumefantrine and amodiaquine against mature gametocytes [36], whilst developing gametocytes appear more susceptible to mefloquine and lumefantrine than to amodiaquine [37]. This would suggest that the maturation of developing gametocytes after initiation of treatment differs between ACT regimens, and this has consequences for post-treatment gametocytaemia.

Contrary to previous studies [38, 39], we found no association between the rate of asexual parasite clearance and gametocytaemia during follow-up. For chloroquine and sulphadoxine-pyrimethamine treatment, posttreatment gametocytaemia and malaria transmission to mosquitoes have been proposed as early parasitological indicators of reduced drug sensitivity $[40,41]$. In our study, $>98 \%$ of all patients cleared their infections by day 2 post-initiation of treatment. Patients subsequently failing treatment were at 15 -fold greater risk of gametocytaemia than those successfully treated, and this was similar for both PCR confirmed recrudescent and new infections. The timing of gametocytaemia coincided with the recurrent asexual parasitaemia. Since the earliest developmental stages of gametocytes are sequestered for 6-8 days in the bone marrow [42], this suggests that gametocyte production started before reappearing asexual parasites were detected by microscopy. The strong association of gametocytaemia with recrudescent infections and new infections warns against a simplistic comparison of treatment regimens based on gametocytaemia shortly after treatment. Initial treatment efficacy and post-treatment prophylaxis that postpones new infection, and therefore de novo gametocyte production, are important determinants of the impact of ACT regimens on malaria transmission.

Whilst our analysis focuses on peripheral gametocytaemia, it is important to acknowledge that this is a surrogate marker of malaria transmission potential. The infectivity of persisting or appearing gametocytes may be affected by the type of antimalarial treatment [9]. Antimalarial drugs may also influence gametocyte sex-ratio [43], which is an important determinant of transmission success, although there is currently no evidence for a differential effect of 
ACT regimens on male and female gametocytes. The only available study that directly determined infectiousness to mosquitoes after ACT regimens compared in this study supports our findings, reporting a two-fold higher mosquito infection rate after DP compared to AL [18], which is consistent with our finding of significantly higher risk of gametocyte appearance after DP (AHR, 2.03; $95 \% \mathrm{CI}$, 1.24-3.34; $P=0.005$ compared to AL). Gametocyte densities commonly fluctuate around the microscopic threshold for detection and the use of molecular gametocyte detection tools would have uncovered a higher proportion of gametocyte carriers [5] at densities capable of contributing to onward malaria transmission [44]. The addition of a single low primaquine dose to ACT can substantially reduce the duration of low density gametocytaemia after treatment [45] and prevent transmission to mosquitoes $[46,47]$ but primaquine is currently not routinely added to ACTs for treatment of uncomplicated malaria. Importantly, although the gametocytocidal properties of first-line ACTs may influence community-wide transmission $[16,48]$, this effect may be modest if transmission is largely driven by asymptomatic individuals who do not seek treatment. The inclusion of these asymptomatically infected individuals in treatment campaigns may have a much larger impact on malaria transmission than the choice of ACT for first-line treatment [6,7].

Our analysis was purposefully restricted to microscopic findings on gametocytaemia, for which most data are available. Although this approach will have missed some gametocyte carriers, this would not affect the comparison of treatment arms. Studies where microscopy, molecular gametocyte data and infectivity results are available indicate that these methods lead to the same conclusions on the comparative effects of antimalarials on post-treatment gametocyte dynamics and infectivity $[15,18]$.

\section{Conclusions}

In conclusion, we identified independent risk factors for the prevalence of gametocytaemia in patients with uncomplicated falciparum malaria in studies conducted on three continents. AS-MQ and AL are superior ACT options in preventing gametocytes shortly after treatment compared to DP or AS-AQ. We hypothesize that this difference is due to the non-artemisinin partner drug defining post-treatment gametocyte dynamics.

\section{Additional files}

Additional file 1: Table S1. Overview of all included studies. ${ }^{1}$ The sensitivity of microscopy methods was classified into one of four categories: 1 = studies in which slides were specifically read for gametocytes, reviewing at least 100 microscopic high power fields or against $\geq 1000$ white blood cells (WBC); $2=$ microscopists specifically instructed to record gametocytes but slides were primarily read for asexual parasites; $\geq 100$ microscopic high power fields per $\geq 1000$ WBC were read; $3=$ microscopists were specifically instructed to record gametocytes; 50-99 microscopic high power fields per 500-999 WBC were read; $4=$ microscopists were not specifically instructed to record gametocytes or the number of examined high power fields was $<50$ or the number of WBC was $<500 .^{2}$ All treatment combinations are loose, unless stated. FDC, fixed dose combination. AS, Artesunate; $M Q$, Mefloquine; AL, Artemether-lumefantrine; DP, Dihydrartemisininpiperaquine; SP, Sulphadoxine-pyrimethamine; $A Q c b, A Q$ co-blisterered loose combination; HL, Halofantrine; QN, Quinine; AM, Artemether, AV, Atovaquone; PG, Proguanil; CQ, Chloroquine; CDA, Chlorproguanildapsone-artesunate; Tet, Tetracycline; $\mathrm{CL}$, Clindamycine. Search strategy: Published prospective trials were identified by the application of the key terms ((malaria OR plasmod*) AND (amodiaquine OR atovaquone OR artemisinin OR arteether OR artesunate OR artemether OR artemotil OR azithromycin OR artekin OR chloroquine OR chlorproguanil OR cycloguanil OR clindamycin OR coartem OR dapsone OR dihydroartemisinin OR duo-cotecxin OR doxycycline OR halofantrine OR lumefantrine OR lariam OR malarone OR mefloquine OR naphthoquine OR naphthoquinone OR piperaquine OR primaquine OR proguanil OR pyrimethamine OR pyronaridine $O R$ quinidine $O R$ quinine $O R$ riamet $O R$ sulphadoxine OR tetracycline OR tafenoquine)) though the PubMed library. Studies on prevention, prophylaxis, review, animal studies or patients with severe malaria were excluded. (DOCX 155 kb)

Additional file 2: Figure S1. Relationship between gametocytaemia on enrolment and baseline haemoglobin concentration, parasitaemia and patient age. The predicted probability of gametocyte carriage at enrolment is plotted from the multivariate model; the line indicates the best fit, the shaded area the $95 \% \mathrm{Cl}$. Only patients from studies with gametocyte detection sensitivity in category 1 or 2 were used for this analysis ( $\geq 100$ high power fields or $\geq 1000$ WBC examined specifically for gametocytes). For the analysis on age, only children $<5$ years of age were included in the analysis. (TIF $480 \mathrm{~kb}$ )

Additional file 3: Table S2. Independent risk factors for the prevalence of gametocytaemia at enrolment in children aged $1-5$ years. Logistic multivariable analysis by region with prevalence of gametocytaemia at enrolment as dependent variable. Nobs, Number of observations; Npos, Number of positive observations. The relationship between gametocyte prevalence at enrolment and age is statistically significant $(P<0.001)$ although not linear, see Additional file 2: Figure S1. Malnutrition (underweight) was not an independent predictor (AOR, 1.11; $95 \% \mathrm{Cl}$, $0.99-1.26 ; P=0.083)$ in Africa and (AOR, 0.92; $95 \% \mathrm{Cl}, 0.47-0.83 ; P=0.823)$ in Asia, after adjustment for age, haemoglobin, parasitaemia and fever (after polynomial transformations, as presented in Additional file 2: Figure S1). (DOC 28 kb)

Additional file 4: Table S3. The effect of treatment dosing on the appearance of gametocytaemia in participants without microscopically detected gametocytaemia before treatment (time to gametocytaemia) and clearance of gametocytaemia in participants with gametocytaemia at enrolment (time to clearance). All analyses of time to clearance are adjusted for log of the initial gametocyte density. Nobs, Number of observations; Npos, Number of positive observations. Under-dosed defined as $\leq 8.4 \mathrm{mg} / \mathrm{kg}$ artemether dose in $\mathrm{AL},<6 \mathrm{mg} / \mathrm{kg}$ dose of artesunate or DHA in other regimens [19]. In the multivariate model estimates are adjusted for other covariates, for time to gametocytaemia: covariates identified in the full final model presented in Table 4; for time to clearance: $A C T$, since no other covariates other than $A C T$ were identified in the final model there were no multivariate models fitted within each $A C T$. $\mathrm{ND}$, No data, HR could not be estimated as there were no patients with gametocytaemia in the under-dose/low-dose group. (DOC 86 kb)

Additional file 5: Table S4. Factors associated with the clearance of gametocytaemia after enrolment in individuals who were gametocytaemic before treatment with artemisinin combination therapy. Nobs, Number of observations; $N$ cleared, Number of patients with day of clearance of gametocytaemia recorded. Derived haemoglobin, conversion from haematocrit: haemoglobin = (haematocrit - 5.62)/2.60 [40]; Anaemia, haemoglobin $<10 \mathrm{~g} / \mathrm{dL}$; Fever, temperature $>37.5^{\circ} \mathrm{C}$; Hyperparasitaemia, parasitaemia $>100,000$ parasites per $\mu \mathrm{L}$; weight-for-age $z$-score, calculated using "igrowup" package developed by WHO [41] in children $<5$ years of 
age: Underweight, weight-for-age $z$-scores $<-2$. Proportional hazards assumption not satisfied for transmission intensity areas, Region, and artemisinin combination therapy. (DOC $59 \mathrm{~kb}$ )

Additional file 6: Table S5. Risk of bias in individual studies included in the analysis. ACT, Artemisinin combination therapy. ${ }^{1}$ For trials with nonACTs, data were only analysed for gametocytaemia on enrolment and regimens, arms, randomization, concealment of treatment, sequence generation and treatment blinding are given as not applicable (NA). ${ }^{2}$ Includes exclusions due to study design (i.e. travellers, repeated episodes). ${ }^{3}$ Evaluated in all patients except for exclusions due to study design or protocol violations. ${ }^{4}$ Evaluated on all included patients treated with ACT and without gametocytaemia on enrolment. ${ }^{5}$ Proportion of patients with time to gametocyte data available but incomplete day 28 follow-up. ${ }^{6}$ Evaluated on all included patients with gametocytaemia on enrolment treated with ACT. ${ }^{7}$ The sensitivity of microscopy methods was classified into one of four categories: $1=$ studies in which slides were specifically read for gametocytes, reviewing at least 100 microscopic high power fields or against $\geq 1000$ white blood cells (WBC); $2=$ microscopists specifically instructed to record gametocytes but slides were primarily read for asexual parasites ; $\geq 100$ microscopic high power fields per $\geq 1000$ WBC were read; $3=$ microscopists were specifically instructed to record gametocytes; 50-99 microscopic high power fields per 500-999 WBC were read; $4=$ microscopists were not specifically instructed to record gametocytes or the number of examined high power fields was $<50$ or the number of WBC was $<500 .{ }^{8}$ No data, no patients with sufficient gametocyte follow-up data that could be included in the analysis. (PDF $408 \mathrm{~kb}$ )

Additional file 7: Table S6. Factors associated with the development of gametocytaemia after enrolment in individuals who were gametocytefree before treatment with artemisinin combination therapy. Cox regression model for time to gametocytaemia. Only patients with complete 28-day follow-up are included. (DOC $35 \mathrm{~kb}$ )

Additional file 8: Table S7. Sensitivity analysis: variation in model coefficients after exclusion of individual studies. ${ }^{1}$ Estimates as obtained in the final multivariate models and listed in main tables. ${ }^{2}$ RSD, Relative standard deviation was calculated as a ratio of standard deviation to mean of the estimates (odds ratio or hazard ratio) calculated by fitting models with one study excluded at a time. (DOC $44 \mathrm{~kb}$ )

Additional file 9: Figure S2. Development of gametocytaemia after treatment evaluated in patients with no gametocytaemia on enrolment and full 28-day follow-up. A: Development of gametocytaemia by artemisinin combination therapy. B: Development of gametocytaemia by treatment outcome. (TIF $284 \mathrm{~kb}$ )

\section{Acknowledgments}

We thank the patients and all the staff who participated in these clinical trials at all the sites and the WorldWide Antimalarial Resistance Network (WWARN) team for technical and administrative support. We would also like to thank Sigma-Tau for sharing data and Hasifa Bukirwa, Marco Corsi, Oumar Faye, Bouasy Hongvanthong, Anand Joshi, Maniphone Khanthavong Moussa Kone, Elfaith Malik, Ushma Mehta, Albert Same-Ekobo, Bhwana Sharma, and Roger CK Tine for their contributions to the studies used in the pooled analysis. WWARN is funded by a Bill and Melinda Gates Foundation grant. The funder did not participate in developing the protocol or writing the paper. Teun Bousema is supported by a fellowship from the European Research Council (ERC-2014-StG 639776).

The members of the WWARN Gametocytes Study Group are the authors of this paper:

Salim Abdulla, Ifakara Health Institute, Dar es Salaam, Tanzania; Jane Achan, Uganda Malaria Surveillance Project, Kampala, Uganda and Medical Research Council Unit, Fajara, The Gambia; Ishag Adam, Faculty of Medicine, University of Khartoum, Khartoum, Sudan; Bereket H Alemayehu, ICAP at Mailman School of Public Health, Columbia University, New York, USA; Richard Allan, The MENTOR Initiative, Crawley, UK; Elizabeth N Allen, Division of Clinical Pharmacology, Department of Medicine, University of Cape Town, Cape Town, South Africa; Anupkumar R Anvikar, National Institute of Malaria Research, New Delhi, India; Emmanuel Arinaitwe, Infectious Diseases Research Collaboration, Kampala, Uganda; Elizabeth A Ashley, Shoklo Malaria
Research Unit, Mahidol-Oxford Tropical Medicine Research Unit, Faculty of Tropical Medicine, Mahidol University, Mae Sot, Thailand and Faculty of Tropical Medicine, Mahidol University, Bangkok, Thailand; Puji Budi Setia Asih, Eijkman Institute for Molecular Biology; Ghulam Rahim Awab, The Mahidol Oxford Tropical Medicine Research Unit (MORU), Bangkok, Thailand and Ministry of Public Health, Islamic Republic of Afghanistan, Kabul, Afghanistan; Karen I Barnes, WWARN, Oxford, UK and Division of Clinical Pharmacology, Department of Medicine, University of Cape Town, Cape Town, South Africa; Quique Bassat, Centro de Investigação em Saude de Manhiça, Manhiça, Mozambique and ISGlobal, Centre de Recerca en Salut Internacional de Barcelona (CRESIB), Hospital Clinic, Universitat de Barcelona, Barcelona, Spain; Elisabeth Baudin, Epicentre, Paris, France; Anders Björkman, Department of Microbiology Tumor and Cell Biology, Karolinska Institutet, Stockholm, Sweden; Francois Bompart, Sanofi, Access to Medicines, Paris, France; Maryline Bonnet, Epicentre, Mbarara, Uganda and Institute de Recherche pour le Developpement UMI233, INSERM U1175, Université de Montpellier, Montelier, France; Steffen Borrmann, Kenya Medical Research Institute/ Wellcome Trust Research Programme, Kilifi, Kenya and Institute for Tropical Medicine, University of Tübingen, Germany; Teun Bousema, Department of Infection and Immunity, London School of Hygiene \& Tropical Medicine (LSHTM), London, UK and Department of Medical Microbiology, Radboud University Nijmegen Medical Centre, Njimegen, The Netherlands; Verena I Carrara, Shoklo Malaria Research Unit, Mahidol-Oxford Tropical Medicine Research Unit, Faculty of Tropical Medicine, Mahidol University, Mae Sot, Thailand and the Mahidol Oxford Tropical Medicine Research Unit (MORU), Bangkok, Thailand; Fabio Cenci, Sigma Tau, Rome, Italy; Francesco Checchi, Epicentre, Paris, France; Michel Cot, IRD, Mother and Child Health in the Tropics Research Unit, Université Paris Descartes, Paris, France; Prabin Dahal, WWARN, Oxford, UK and Centre for Tropical Medicine and Global Health, Nuffield Department of Clinical Medicine, University of Oxford, Oxford, UK; Umberto D'Alessandro, Medical Research Council Unit, Fajara, The Gambia, LSHTM, London, UK and Institute of Tropical Medicine, Antwerp, Belgium; Philippe Deloron, Institut de Recherche pour le Développement, Mother and Child Faced with Tropical Infections Research Unit, Paris, France and PRES Paris Sorbonne Cité, Université Paris Descartes, Paris, France; Abdoulaye Djimde, Malaria Research and Training Center, Department of Epidemiology of Parasitic Diseases, Faculty of Medicine, Pharmacy and OdontoStomatology, University of Bamako, Bamako, Mali; Arjen Dondorp, The Mahidol Oxford Tropical Medicine Research Unit (MORU), Bangkok, Thailand Centre for Tropical Medicine and Global Health, Nuffield Department of Clinical Medicine, University of Oxford, Oxford, UK; Grant Dorsey, Department of Medicine, University of California San Francisco, San Francisco, USA; Ogobara K Doumbo, Malaria Research and Training Center, Department of Epidemiology of Parasitic Diseases, Faculty of Medicine, Pharmacy and Odonto-Stomatology, University of Bamako, Bamako, Mali; Chris J Drakeley, Department of Infection and Immunity, LSHTM, London, UK; Stephan Duparc, Medicines for Malaria Venture, Geneva, Switzerland; Emmanuelle Espie, Epicentre, Paris, France; Abul Faiz, Dev Care Foundation, Bangladesh; Catherine O Falade, Department of Pharmacology and Therapeutics, College of Medicine, University of Ibadan, Ibadan, Nigeria; Caterina Fanello, The Mahidol Oxford Tropical Medicine Research Unit (MORU), Bangkok, Thailand; Jean-François Faucher, Institut de Recherche pour le Développement (IRD), Mother and Child Health in the Tropics Research Unit, Paris, France and Faculté de Pharmacie, Université Paris Descartes, Paris, France and Department of Infectious Diseases, Besançon University Medical Center, Besançon, France; Babacar Faye, Department of Medical Parasitology, Medical Faculty, Université Cheikh Anta Diop, Dakar, Senegal; Scott Filler, The Global Fund to Fight AIDS, Tuberculosis and Malaria, Geneva, Switzerland; Bakary Fofana, Malaria Research and Training Center, Department of Epidemiology of Parasitic Diseases, Faculty of Medicine, Pharmacy and OdontoStomatology, University of Bamako, Bamako, Mali; Carole Fogg, University of Portsmouth, Portsmouth, UK; Adama Gansane, Centre National de Recherche et de Formation sur le Paludisme, Ouagadougou, Burkina Faso; Oumar Gaye, Department of Medical Parasitology, Medical Faculty, Université Cheikh Anta Diop, Dakar, Senegal; Blaise Genton, Department of Epidemiology and Public Health, Swiss Tropical and Public Health Institute, Basel, Switzerland and Division of Infectious Diseases and Department of Ambulatory Care and Community Medicine, University Hospital, Lausanne, Switzerland; Peter W Gething, Spatial Ecology and Epidemiology Group, Department of Zoology, University of Oxford, Oxford, UK; Raquel Gonzalez, Centro de Investigação em Saude de Manhiça, Manhiça, Mozambique and ISGlobal, Barcelona Ctr. 
Int Health Res. (CRESIB), Hospital Clínic, Universitat de Barcelona, Spain; Francesco Grandesso, Epicentre, Paris, France; Brian Greenwood, Department of Diseases Control, LSHTM, London, UK; Anastasia Grivoyannis, University of Washington, USA; Philippe J Guerin, WWARN, Oxford, UK and Centre for Tropical Medicine and Global Health, Nuffield Department of Clinical Medicine, University of Oxford, Oxford, UK; Kamal Hamed, Novartis Pharmaceuticals Corporation, East Hanover, USA; Christoph Hatz, Medical Department, Swiss Tropical Institute, Basel, Switzerland; Simon I Hay, Wellcome Trust Centre for Human Genetics, University of Oxford, Oxford, UK and Institute for Health Metrics and Evaluation, University of Washington, Seattle, USA; Eva Maria Hodel, Swiss Tropical Institute and Public Health Institute, Basel, Switzerland and Clinical Sciences, Liverpool School of Tropical Medicine, Liverpool, UK; Georgina S Humphreys, WWARN, Oxford, UK and Centre for Tropical Medicine and Global Health, Nuffield Department of Clinical Medicine, University of Oxford, Oxford, UK; Jimee Hwang, U.S. Centers for Disease Control and Prevention, Atlanta, USA and Global Health Group, University of California San Francisco, San Francisco, USA; Bart Janssens, Médecins Sans Frontières, Operational Centre Brussels, Brussels, Belgium; Daddi Jima, Federal Ministry of Health, Addis Ababa, Ethiopia; Elizabeth Juma, Kenya Medical Research Institute, Nairobi, Kenya; S Patrick Kachur, U.S. Centers for Disease Control and Prevention, Atlanta, USA; Piet Kager, Academic Medical Centre, Amsterdam, Netherlands; Moses R Kamya, Makerere University College of Health Sciences, Kampala, Uganda; Melissa Kapulu, Kenya Medical Research Institute/Wellcome Trust Research Programme, Kilifi, Kenya and Centre for Tropical Medicine and Global Health, Nuffield Department of Clinical Medicine, University of Oxford, Oxford, UK; Corine Karema, Malaria \& Other Parasitic Diseases Division, RBC, Ministry of Health, Kigali, Rwanda; Kassoum Kayentao, Malaria Research and Training Centre, Department of Epidemiology of Parasitologic Diseases, Faculty of Medicine, Pharmacy and Dentistry, University of Bamako, Mali; Jean R Kiechel, Drugs for Neglected Diseases initiative (DNDi), Geneva, Switzerland; Poul-Erik Kofoed, Projecto de Saúde de Bandim, Bissau, Guinea-Bissau and Health Services Research Unit, Lillebaelt Hospital/IRS University of Southern Denmark, Vejle, Denmark and Department of Paediatrics, Kolding Hospital, Kolding, Denmark; Valerie Lameyre, Sanofi, Access to Medicines, Paris, France; Sue J Lee, Faculty of Tropical Medicine, Mahidol University, Bangkok, Thailand and Centre for Tropical Medicine and Global Health, Nuffield Department of Clinical Medicine, University of Oxford, Oxford, UK; Bertrand Lell, Institute for Tropical Medicine, University of Tubingen, Tubingen, Germany and Centre de Recherches Médicales de Lambaréné, Lambaréné, Gabon; Nines Lima, Médecins Sans Frontières - Operational Centre Barcelona Athens, Barcelona, Spain; Kevin Marsh, Centre for Tropical Medicine and Global Health, Nuffield Department of Clinical Medicine, University of Oxford, Oxford, UK and Kenya Medical Research Institute/Wellcome Trust Research Programme, Kilifi, Kenya; Andreas Mårtensson, Department of Microbiology, Tumor and Cell Biology, Karolinska Institutet, Stockholm, Sweden and Department of Women's and Children's Health, International Maternal and Child Health (IMCH), Upssala University, Uppsala, Sweden; Achille Massougbodji, Centre d'Etudes et de Recherche sur le Paludisme Associé à la Grossesse et à l'Enfant (CERPAGE), Faculté des Sciences de la Santé (FSS), Université d'Abomey-Calavi, Cotonou, Bénin; Mayfong Mayxay, Lao-OxfordMahosot Hospital, Wellcome Trust Research Unit (LOMWRU), Microbiology Laboratory, Mahosot Hospital, Vientiane, Lao PDR and Faculty of Postgraduate Studies, University of Health Sciences, Vientiane, Lao PDR and Centre for Tropical Medicine and Global Health, Nuffield Department of Clinical Medicine, University of Oxford, Oxford, UK; Rose McGready, Shoklo Malaria Research Unit, Mahidol-Oxford Tropical Medicine Research Unit, Faculty of Tropical Medicine, Mahidol University, Mae Sot, Thailand and Centre for Tropical Medicine and Global Health, Nuffield Department of Clinical Medicine, University of Oxford, Oxford, UK; Hervé Menan, Department of Parasitology, Faculty of Pharmacy, University of Cocody, Abidjan, Côte d'Ivoire; Clara Menendez, Barcelona Institute for Global Health (ISGlobal) Hospital Clinic, University of Barcelona, Spain and CISM, Manhiça Health Research Center, Manhiça, Mozambique; Petra Mens, Royal Tropical Institute, KIT Biomedical Research, Amsterdam, The Netherlands and Division of Infectious Diseases, Center for Tropical Medicine \& Travel Medicine, Academic Medical Center, University of Amsterdam, The Netherlands; Martin Meremikwu, Department of Paediatrics, University of Calabar and Nigeria Institute of Tropical Diseases Research \& Prevention, Calabar, Nigeria; Frank P Mockenhaupt, Institute of Tropical Medicine and International Health, Charite-Universitatsmedizin Berlin, Germany; Clarissa Moreira, WWARN,
Oxford, UK and Centre for Tropical Medicine and Global Health, Nuffield Department of Clinical Medicine, University of Oxford, Oxford, UK; Carolyn Nabasumba, Epicentre, Paris, France and Faculty of Medicine, Mbarara University of Science and Technology, Mbarara, Uganda; Michael Nambozi, Tropical Diseases Research Centre, Ndola, Zambia; Jean-Louis Ndiaye, Parasitology and Mycology Laboratory, Medical Faculty, Université Cheikh Anta Diop, Dakar, Senegal; Paul N Newton, Lao-Oxford-Mahosot HospitalWellcome Trust Research Unit, Mahosot Hospital, Vientiane, Lao PDR and Centre for Tropical Medicine and Global Health, Nuffield Department of Clinical Medicine, University of Oxford, Oxford, UK; Billy E Ngasala, Department of Parasitology, Muhimbili University of Health and Allied Sciences, Dar es Salaam, Tanzania and Malaria Research, Infectious Disease Unit, Department of Medicine, Solna, Karolinska Institutet, Stockholm, Sweden; Francois Nosten, Shoklo Malaria Research Unit, Mahidol-Oxford Tropical Medicine Research Unit, Faculty of Tropical Medicine, Mahidol University, Mae Sot, Thailand and Centre for Tropical Medicine and Global Health, Nuffield Department of Clinical Medicine, University of Oxford, Oxford, UK; Christian Nsanzabana, WWARN, Oxford, UK and Centre for Tropical Medicine and Global Health, Nuffield Department of Clinical Medicine, University of Oxford, Oxford, UK; Andre Toure Offianan, Malariology Department, Institut Pasteur, Abidjan, Côte d'Ivoire; Mary Oguike, Department of Immunology \& Infection, LSHTM, London, UK; Bernhards R Ogutu, Kenya Medical Research Institute/United States Army Medical Research Unit, Kisumu, Kenya; Piero Olliaro, UNICEF/UNDP/World Bank/WHO Special Programme for Research and Training in Tropical Diseases (WHO/TDR), Geneva, Switzerland; Sabah A Omar, International Centre for Insect Physiology and Ecology (ICIPE), Mbita Point, Kenya; Lyda Osorio, Facultad de Salud, Universidad del Valle, Cali, Colombia; Seth Owusu-Agyei, Kintampo Health Research Centre, Kintampo, Ghana; Louis K Penali, WWARN, Dakar, Senegal; Mbaye Pene, Department of Medical Parasitology, Medical Faculty, Université Cheikh Anta Diop, Dakar, Senegal; Judy Peshu, Kenya Medical Research Institute/Wellcome Trust Research Programme, Kilifi, Kenya; Patrice Piola, Institut Pasteur de Madagascar, Antananarivo, Madagascar; Zul Premji, Muhimbili University of Health and Allied Sciences, Dar es Salaam, Tanzania; Ric N Price, Menzies School of Health Research and Charles Darwin University, Darwin, Australia and Centre for Tropical Medicine and Global Health, Nuffield Department of Clinical Medicine, University of Oxford, Oxford, UK and WWARN, Oxford, UK; Michael Ramharter, Department of Medicine I, Division of Infectious Diseases and Tropical Medicine, Medical University Vienna, Austria and Institut für Tropenmedizin, Universität Tübingen, Germany and Centre de Recherches Medicales de Lambaréné, Gabon; Lars Rombo, Infectious Diseases Unit, Department of Medicine Solna, Karolinska University Hospital, Karolinska Institutet, Stockholm, Sweden; Cally Roper, LSHTM, London, UK; Philip J Rosenthal, Department of Medicine, University of California San Francisco, San Francisco, USA; Issaka Sagara, Malaria Research and Training Center, Department of Epidemiology of Parasitic Diseases, Faculty of Medicine, Pharmacy and Odonto-Stomatology, University of Bamako, Bamako, Mali; Patrick Sawa, Human Health Division, International Centre for Insect Physiology and Ecology, Mbita, Kenya; Henk DFH Schallig, Royal Tropical Institute, KIT Biomedical Research, Amsterdam, The Netherlands; Birgit Schramm, Epicentre, Paris, France; Seif A Shekalaghe, Ifakara Health Institute, Bagamoyo, Tanzania; Carol H Sibley, WWARN, Oxford, UK and Department of Genome Sciences, University of Washington, Seattle, USA; Sodiomon Sirima, Centre National de Recherche et de Formation sur le Paludisme, Ouagadougou, Burkina Faso; Frank Smithuis, Myanmar Oxford Clinical Research Unit (MOCRU), Yangon, Myanmar and Medical Action Myanmar, Yangon, Myanmar; Doudou Sow, Service de Parasitologie, Medical Faculty, Université Cheikh Anta Diop, Dakar, Sénégal; Sarah G Staedke, Department of Clinical Research, LSHTM, London, UK and Infectious Disease Research Collaboration, Kampala, Uganda; Kasia Stepniewska, WWARN, Oxford, UK and Centre for Tropical Medicine and Global Health, Nuffield Department of Clinical Medicine, University of Oxford, Oxford, UK; Inge Sutanto, Department of Parasitology, Faculty of Medicine, University of Indonesia, Jakarta, Indonesia; Colin J Sutherland, Department of Immunology \& Infection, LSHTM, London, UK; Todd D Swarthout, Médecins Sans Frontières, London, UK; Din Syafruddin, Eijkman Institute for Molecular Biology, Jakarta, Indonesia; Khadime Sylla, Service de Parasitologie-Mycologie Médicale, Université Cheikh Anta Diop, Dakar, Senegal; Ambrose O Talisuna, University of Oxford/KEMRI/Wellcome Trust Research Programme, Nairobi, Kenya; Walter R Taylor, Institute for Biomechanics, Department of Health Science and Technology, Eidgenössische Technische Hochschule Zürich, 
Zürich, Switzerland; Emmanuel A Temu, The MENTOR Initiative, Crawley, UK and Swiss Tropical and Public Health Institute, Basel, Switzerland and University of Basel, Basel, Switzerland; Feiko Ter Kuile, Liverpool School of Tropical Medicine, Liverpool, UK and Kenya Medical Research Institute (KEMRI), Centre for Global Health Research, Kisumu, Kenya; Halidou Tinto, Institut de Recherce en Sciences de la Sante, Bobo Dioulasso, Burkina Faso and Centre Muraz, Bobo Dioulasso, Burkina Faso; Emiliana Tjitra, National Institute of Health Research and Development, Ministry of Health, Jakarta, Indonesia; Johan Ursing, Projecto de Saúde de Bandim, Indepth Network, Bissau, Guinea-Bissau and Department of Microbiology, Tumor and Cell Biology, Karolinska Institutet, Stockholm, Sweden; Neena Valecha, National Institute of Malaria Research, New Delhi, India; Ingrid van den Broek, Médecins Sans Frontières, London, UK and Centre for Infectious Disease Control, National Institute for Public Health and the Environment, Bilthoven, The Netherlands; Michel van Herp, Médecins Sans Frontières, Operational Centre Brussels, Brussels, Belgium; Michele van Vugt, Division of Infectious Diseases, Center for Tropical Medicine \& Travel Medicine, Academic Medical Center, University of Amsterdam, The Netherlands; Stephen A Ward, Department of Parasitology, Liverpool School of Tropical Medicine, Liverpool, UK; Nicholas J White, Faculty of Tropical Medicine, Mahidol University, Bangkok, Thailand and Centre for Tropical Medicine and Global Health, Nuffield Department of Clinical Medicine, University of Oxford, Oxford, UK; Peter A Winstanley, School of Clinical Sciences, University of Liverpool, Liverpool, UK; Charles J Woodrow, The Mahidol Oxford Tropical Medicine Research Unit (MORU), Bangkok, Thailand and Centre for Tropical Medicine and Global Health, Nuffield Department of Clinical Medicine, University of Oxford, Oxford, UK; Adoke Yeka, Uganda Malaria Surveillance Project, Kampala, Uganda; Julien Zwang, Drugs for Neglected Diseases initiative (DNDi), Geneva, Switzerland.

\section{Authors' contributions}

TB, KSt, GSH, CJD, CHS, PJG and RNP conceived and designed the experiments. KSt and GSH analyzed the pooled individual patient data. GSH, CNs and PDa performed literature search. GSH, PDa and CMo data management. SA, JA, IA, BHA, RA, ENA, ARA, EA, EAA, PBSA, GRA, KIB, QB, EB, AB, $F B, M B, S B, T B, V I C, F C$, FCh, MC, UdA, PDe, ADj, ADo, GD, OKD, CJD, SD, EE, AF, $\mathrm{COF}, \mathrm{CFa}$, JFF, BFa, SF, BFo, CFo, AGa, OG, BGe, PWG, RG, FG, BGr, AGr, PJG, KH, $\mathrm{CH}, \mathrm{SIH}, \mathrm{EMH}, J \mathrm{H}, \mathrm{BJ}, \mathrm{DJ}, \mathrm{EJ}, \mathrm{SPK}, \mathrm{PK}, \mathrm{MRK}, \mathrm{MK}, \mathrm{CK}, \mathrm{KK}, J R K$, PEK, VL, SJL, BL, NL, KM, AMar, AMas, MMa, RM, HM, CMe, PM, MMe, FPM, CNa, MN, JLN, PNN, BEN, FN, CNs, ATO, MO, BRO, PO, SAO, LO, SOA, LKP, MP, JP, PP, ZP, RNP, MR, LR, CR, PJR, ISa, PS, HDFHS, BS, SAS, SS, FS, DSo, SGS, KSt, ISu, CJS, TDS, DSy, KSy, AOT, WRT, EAT, FTK, HT, ET, JU, NV, IvdB, MvH, MvV, SAW, NJW, PAW, CJW, AY and JZ performed the original experiments. PWG and SIH provided transmission intensity estimates. TB, KSt, GSH, CJD, CHS, PJG and RNP wrote the first draft of the manuscript. SA, JA, IA, BHA, RA, ENA, ARA, EA, EAA, PBSA, GRA, KIB, QB, EB, $A B, F B, M B, S B, T B, V I C, F C$, FCh, MC, PDa, UdA, PDe, ADj, ADo, GD, OKD, CJD, SD, $E E, A F, C O F, C F a, J F F, B F a, S F, B F o, C F o, A G a, O G, B G e, P W G, R G, F G, B G r, A G r$, PJG, KH, CH, SIH, EMH, GSH, JH, BJ, DJ, EJ, SPK, PK, MRK, MK, CK, KK, JRK, PEK, VL, SJL, BL, NL, KM, AMar, AMas, MMa, RM, HM, CMe, PM, MMe, FPM, CMo, CNa, MN, JLN, PNN, BEN, FN, CNS, ATO, MO, BRO, PO, SAO, LO, SOA, LKP, MP, JP, PP, ZP, RNP, MR, LR, CR, PJR, ISa, PS, HDFHS, BS, SAS, CHS, SS, FS, Dso, SGS, KSt, ISu, CJS, TDS, Dsy, KSY, AOT, WRT, EAT, FTK, HT, ET, JU, NV, IvdB, MvH, MvV, SAW, NJW, PAW, CJW, AY and JZ International Committee of Medical Journal Editors (ICMJE) criteria for authorship read and met. All authors contributed to the writing of the manuscript and agree with manuscript results and conclusions. All authors read and approved the final manuscript.

\section{Competing interests}

FB and VL are employees of Sanofi. SD is an employee of Medicines for Malaria Venture, Geneva, Switzerland. KH is an employee of Novartis Pharmaceuticals, East Hanover, NJ, USA. FS is an employee of Sigma Tau. The remaining authors declare that no competing interests exist.

\section{Received: 5 February 2016 Accepted: 27 April 2016 Published online: 24 May 2016}

\section{References}

1. World Health Organization. World Malaria Report 2014. Geneva: WHO; 2014.

2. Bhatt S, Weiss DJ, Cameron E, Bisanzio D, Mappin B, Dalrymple U, et al. The effect of malaria control on Plasmodium falciparum in Africa between 2000 and 2015. Nature. 2015;526(7572):207-11.
3. Griffin JT, Hollingsworth TD, Okell LC, Churcher TS, White M, Hinsley W, Bousema T, Drakeley CJ, Ferguson NM, Basanez MG, et al. Reducing Plasmodium falciparum malaria transmission in Africa: a model-based evaluation of intervention strategies. PLoS Med. 2010;7(8).

4. Price R, Nosten F, Simpson JA, Luxemburger C, Phaipun L, ter Kuile F, et al. Risk factors for gametocyte carriage in uncomplicated falciparum malaria. Am J Trop Med Hyg. 1999;60(6):1019-23.

5. Bousema T, Drakeley C. Epidemiology and infectivity of Plasmodium falciparum and Plasmodium vivax gametocytes in relation to malaria control and elimination. Clin Mirobiol Rev. 2011;24(2):377-410.

6. Slater HC, Ross A, Ouedraogo AL, White L, Nguon C, Walker PG, et al. Assessing the impact of next-generation rapid diagnostic tests on Plasmodium falciparum malaria elimination strategies. Nature. 2015; 528(7580):S94-101.

7. Johnston GL, Gething PW, Hay SI, Smith DL, Fidock DA. Modeling withinhost effects of drugs on Plasmodium falciparum transmission and prospects for malaria elimination. PLoS Comp Biol. 2014:10(1):e1003434.

8. Bousema T, Okell L, Shekalaghe S, Griffin JT, Omar S, Sawa P, et al. Revisiting the circulation time of Plasmodium falciparum gametocytes: molecular detection methods to estimate the duration of gametocyte carriage and the effect of gametocytocidal drugs. Malar J. 2010;9:136.

9. Targett G, Drakeley C, Jawara M, von Seidlein L, Coleman R, Deen J, et al. Artesunate reduces but does not prevent posttreatment transmission of Plasmodium falciparum to Anopheles gambiae. J Infect Dis. 2001;183(8):1254-9.

10. WorldWide Antimalarial Resistance Network DPSG. The effect of dosing regimens on the antimalarial efficacy of dihydroartemisinin-piperaquine: a pooled analysis of individual patient data. PLoS Med. 2013;10(12):e1001564. Discussion e1001564.

11. White NJ. Assessment of the pharmacodynamic properties of antimalarial drugs in vivo. Antimicrob Ag Chemother. 1997;41 (7):1413-22.

12. Adjalley SH, Johnston GL, Li T, Eastman RT, Ekland EH, Eappen AG, et al. Quantitative assessment of Plasmodium falciparum sexual development reveals potent transmission-blocking activity by methylene blue. Proc Natl Acad Sci U S A. 2011;108(47):E1214-23.

13. Price RN, Nosten F, Luxemburger $C$, ter Kuile FO, Paiphun $L$, Chongsuphajaisiddhi T, et al. Effects of artemisinin derivatives on malaria transmissibility. Lancet. 1996:347(9016):1654-8.

14. Sutherland CJ, Ord R, Dunyo S, Jawara M, Drakeley CJ, Alexander N, et al. Reduction of malaria transmission to Anopheles mosquitoes with a six-dose regimen of co-artemether. PLoS Med. 2005;2(4):e92.

15. Bousema JT, Schneider P, Gouagna LC, Drakeley CJ, Tostmann A, Houben R, et al. Moderate effect of artemisinin-based combination therapy on transmission of Plasmodium falciparum. J Infect Dis. 2006;193(8):1151-9.

16. Nosten F, van Vugt M, Price R, Luxemburger C, Thway KL, Brockman A, et al. Effects of artesunate-mefloquine combination on incidence of Plasmodium falciparum malaria and mefloquine resistance in western Thailand: a prospective study. Lancet. 2000;356(9226):297-302.

17. Barnes Kl, Durrheim DN, Little F, Jackson A, Mehta U, Allen E, et al. Effect of artemether-lumefantrine policy and improved vector control on malaria burden in KwaZulu-Natal. South Africa PLoS Med. 2005;2(11):e330.

18. Sawa P, Shekalaghe SA, Drakeley CJ, Sutherland CJ, Mweresa CK, Baidjoe AY, et al. Malaria transmission after artemether-lumefantrine and dihydroartemisinin-piperaquine: a randomized trial. J Infect Dis. 2013;207(11):1637-45.

19. Four Artemisinin-Based Combinations Study Group. A head-to-head comparison of four artemisinin-based combinations for treating uncomplicated malaria in African children: a randomized trial. PLoS Med. 2011;8(11):e1001119.

20. WorldWide Antimalarial Resistance Network. Gametocyte Carriage Study Group Statistical Analysis Plan. 2015. http://www.wwarn.org/toolsresources/gametocyte-carriage-study-group-statistical-analysis-plan. Accessed 20 May 2014

21. Gething PW, Patil AP, Smith DL, Guerra CA, Elyazar IR, Johnston GL, et al. A new world malaria map: Plasmodium falciparum endemicity in 2010. Malar J. 2011;10:378

22. Royston P, Altman DG. Regression using fractional polynomials of continuous covariates: parsimonious parametric modelling. Appl Stat. 1994;43:429-67.

23. World Health Organization. Guidelines for the treatment of malaria. 2nd ed. Geneva: WHO; 2010. 
24. Stepniewska K, Price RN, Sutherland CJ, Drakeley CJ, von Seidlein L, Nosten F, et al. Plasmodium falciparum gametocyte dynamics in areas of different malaria endemicity. Malar J. 2008;7:249.

25. Price RN, Simpson JA, Nosten F, Luxemburger C, Hkirjaroen L, ter Kuile F, et al. Factors contributing to anemia after uncomplicated falciparum malaria. Am J Trop Med Hyg. 2001;65(5):614-22.

26. Taylor WR, Widjaja H, Basri H, Tjitra E, Ohrt C, Taufik T, et al. Haemoglobin dynamics in Papuan and non-Papuan adults in northeast Papua, Indonesia with acute, uncomplicated vivax or falciparum malaria. Malar J. 2013;12:209.

27. Sowunmi A, Fateye BA, Adedeji AA, Fehintola FA, Happi TC. Risk factors for gametocyte carriage in uncomplicated falciparum malaria in children. Parasitol. 2004;129(Pt 3):255-62.

28. Trager W, Gill GS, Lawrence C, Nagel RL. Plasmodium falciparum: enhanced gametocyte formation in vitro in reticulocyte-rich blood. Exp Parasitol. 1999:91(2):115-8.

29. Reece SE, Duncan AB, West SA, Read AF. Host cell preference and variable transmission strategies in malaria parasites. Proc Biol Sci. 2005;272(1562):511-7.

30. Akim NI, Drakeley C, Kingo T, Simon B, Senkoro K, Sauerwein RW. Dynamics of P. falciparum gametocytemia in symptomatic patients in an area of intense perennial transmission in Tanzania. Am J Trop Med Hyg. 2000;63(3-4):199-203

31. Nacher M, Singhasivanon P, Silachamroon U, Treeprasertsuk S, Tosukhowong T, Vannaphan S, et al. Decreased hemoglobin concentrations, hyperparasitemia, and severe malaria are associated with increased Plasmodium falciparum gametocyte carriage. J Parasitol. 2002:88(1):97-101.

32. Bassat Q, Mulenga M, Tinto H, Piola P, Borrmann S, Menendez C, et al. Dihydroartemisinin-piperaquine and artemether-lumefantrine for treating uncomplicated malaria in African children: a randomised, non-inferiority trial. PLoS One. 2009;4(11):e7871.

33. Yeka A, Dorsey G, Kamya MR, Talisuna A, Lugemwa M, Rwakimari JB, et al. Artemether-lumefantrine versus dihydroartemisinin-piperaquine for treating uncomplicated malaria: a randomized trial to guide policy in Uganda. PLoS One. 2008;3(6):e2390.

34. Zwang J, Ashley EA, Karema C, D'Alessandro U, Smithuis F, Dorsey G, et al. Safety and efficacy of dihydroartemisinin-piperaquine in falciparum malaria: a prospective multi-centre individual patient data analysis. PLoS One. 2009;4(7):e6358

35. World Health Organization. Guidelines for the treatment of malaria. 3rd ed. Geneva: WHO; 2015.

36. Bolscher JM, Koolen KM, van Gemert GJ, van de Vegte-Bolmer MG, Bousema T, Leroy D, et al. A combination of new screening assays for prioritization of transmission-blocking antimalarials reveals distinct dynamics of marketed and experimental drugs. J Antimicrob Chemother. 2015;70:1357-66.

37. Lucantoni L, Duffy S, Adjalley SH, Fidock DA, Avery VM. Identification of MMV malaria box inhibitors of plasmodium falciparum early-stage gametocytes using a luciferase-based high-throughput assay. Antimicrob Ag Chemother. 2013;57(12):6050-62.

38. Ashley EA, Dhorda M, Fairhurst RM, Amaratunga C, Lim P, Suon S, et al. Spread of artemisinin resistance in Plasmodium falciparum malaria. N Engl J Med. 2014;371(5):411-23

39. Carrara VI, Zwang J, Ashley EA, Price RN, Stepniewska K, Barends M, et al. Changes in the treatment responses to artesunate-mefloquine on the northwestern border of Thailand during 13 years of continuous deployment. PLoS One. 2009;4(2):e4551.

40. Barnes Kl, Little F, Mabuza A, Mngomezulu N, Govere J, Durrheim D, et al. Increased gametocytemia after treatment: an early parasitological indicator of emerging sulfadoxine-pyrimethamine resistance in falciparum malaria. J Infect Dis. 2008;197(11):1605-13.

41. Hallett RL, Dunyo S, Ord R, Jawara M, Pinder M, Randall A, et al. Chloroquine/sulphadoxine-pyrimethamine for gambian children with malaria: transmission to mosquitoes of multidrug-resistant Plasmodium falciparum. PLoS Clin Trials. 2006;1(3):e15.

42. Joice R, Nilsson SK, Montgomery J, Dankwa S, Egan E, Morahan B, et al. Plasmodium falciparum transmission stages accumulate in the human bone marrow. Sci Transl Med. 2014;6(244):244re245.

43. Delves MJ, Ruecker A, Straschil U, Lelievre J, Marques S, Lopez-Barragan MJ, et al. Male and female Plasmodium falciparum mature gametocytes show different responses to antimalarial drugs. Antimicr Ag Chemother. 2013:57(7):3268-74.
44. Churcher TS, Bousema T, Walker M, Drakeley C, Schneider P, Ouedraogo AL, et al. Predicting mosquito infection from Plasmodium falciparum gametocyte density and estimating the reservoir of infection. eLife. 2013;2: e00626.

45. Eziefula AC, Bousema T, Yeung S, Kamya M, Owaraganise A, Gabagaya G, et al. Single dose primaquine for clearance of Plasmodium falciparum gametocytes in children with uncomplicated malaria in Uganda: a randomised, controlled, double-blind, dose-ranging trial. Lancet Infect Dis. 2014;14(2):130-9.

46. White NJ. Primaquine to prevent transmission of falciparum malaria. Lancet Infect Dis. 2013;13(2):175-81.

47. Dicko A, Brown JM, Diawara H, Baber I, Mahamar A, Soumare HM, Sanogo K, Koita F, Keita S, Traore SF, et al. Primaquine to reduce transmission of Plasmodium falciparum malaria in Mali: a single-blind, dose-ranging, adaptive randomised phase 2 trial. Lancet Infect Dis. 2016.

48. Okell LC, Cairns M, Griffin JT, Ferguson NM, Tarning J, Jagoe G, et al. Contrasting benefits of different artemisinin combination therapies as first-line malaria treatments using model-based cost-effectiveness analysis. Nat Comm. 2014:5:5606.

\section{Submit your next manuscript to BioMed Central and we will help you at every step:}

- We accept pre-submission inquiries

- Our selector tool helps you to find the most relevant journal

- We provide round the clock customer support

- Convenient online submission

- Thorough peer review

- Inclusion in PubMed and all major indexing services

- Maximum visibility for your research

Submit your manuscript at www.biomedcentral.com/submit
) Biomed Central 